\title{
Recent Advancements and Applications of Inhalable Microparticles Based Drug Delivery Systems in Respiratory Disorders
}

\author{
Neha Kanojia $1(\mathbb{D}$, Sukhbir Singh $2 \mathbb{( D )}$, Jitender Singh $3(\mathbb{D}$, Neelam Sharma 2,* $\mathbb{D}$, Ajmer Singh \\ Grewal ${ }^{1}$ (D) , Lata Rani ${ }^{1}$ (D), Komal Thapa ${ }^{1}$ (D), Sandeep Arora 2 (D)

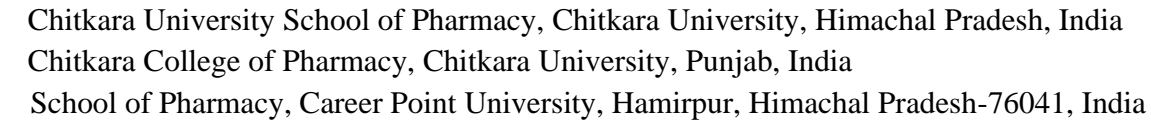

Scopus Author ID 57214054056

Received: 17.09.2020; Revised: 7.10.2020; Accepted: 9.10.2020; Published: 12.10.2020

\begin{abstract}
Lower respiratory infections are the third leading cause of death, as stated by the world health organization. The pulmonary route requires reduced dose, exhibits immediate drug release, reduced first-pass hepatic metabolism and adverse effects, and systemic drug release. Therefore, an overview of recent advancements in inhalable microparticles reported in publications and patents published in the last few years has been summarized. Recent innovations in inhaler technology and major challenges in pulmonary drug delivery have been discussed briefly. The analysis was collected through comprehensive literature searches from databases such as PubMed, Google Scholar, and ScienceDirect. The recent patents on inhalable microparticles have been compiled through the PATENTSCOPE database via WIPO website. The keywords used in the search strategy were 'inhalable microparticles', 'polymeric microparticles', 'large porous microparticles', 'solid lipid microparticles', 'cyclodextrin complex microparticles', 'respiratory disorders', 'patent, 'inhaler technology', 'drug deposition', 'pharmacokinetic processes' and 'pulmonary drug delivery' in various combinations. A survey of literature revealed that 44 publications and 14 patents, and 9 recent innovations in inhaler technology had been reported regarding inhalable microparticles in respiratory disorders. This review briefly recapitulates the pharmacokinetic processes involved in the pulmonary drug delivery route, mechanisms of drug deposition in the respiratory tract, types, and production methodology of inhalable microparticles.
\end{abstract}

Keywords: cyclodextrin complex microparticles; drug deposition; inhalable microparticles; large porous microparticles; polymeric microparticles; respiratory disorders; solid lipid microparticles.

(C) 2020 by the authors. This article is an open-access article distributed under the terms and conditions of the Creative Commons Attribution (CC BY) license (https://creativecommons.org/licenses/by/4.0/).

\section{Introduction}

Lower respiratory infections are the third leading cause of death, as stated by the world health organization. Lungs have huge surface area for absorption (140 m2) and thin (0.1-0.2 $\mu \mathrm{m})$ absorption mucosal membrane, therefore, lungs play a vital role in the gaseous exchange and oxygen supply to all the cells. Therefore, the pulmonary route has the potential for noninvasive administration for local and systemic drug delivery in the respiratory tract owing to the larger alveolar surface area, rich blood supply, thin blood alveolar barrier, and high permeability [1-3]. This route requires reduced dose, exhibits immediate drug release, reduced first-pass hepatic metabolism and adverse effects, and systemic drug release [4]. Hence, the 
pulmonary drug delivery route has gained attention as a preferred route of drug administration to cure several respiratory disorders, as displayed in Figure 1. The drug targeting approaches consist of direct drug delivery into the lungs, primarily through inhalation medication exploiting dry powder inhalers. The disadvantages of the pulmonary route include complexity in pulmonary drug devices, diminutive duration of action owing to fast removal of active ingredients from lungs, inadequate drug absorption due to physical barriers, and local side effects attributable to pharyngeal deposition [5]. Inhalable microparticles have appeared as considerable development for the management of respiratory disorders on account of improved drug's therapeutic index, prolonged biological half-life, and reduced toxicity [6-8].

For a compilation of this review, the literature was collected from databases such as PubMed, Google Scholar, and ScienceDirect. The recent patents on inhalable microparticles for respiratory disorders have been compiled through the PATENTSCOPE database via WIPO website. The keywords used in the search strategy were inhalable microparticles', 'polymeric microparticles', 'large porous microparticles', 'solid lipid microparticles', 'cyclodextrin complex microparticles', 'respiratory disorders', 'patent, 'inhaler technology', 'drug deposition', 'pharmacokinetic processes' and 'pulmonary drug delivery' in various combinations. The present review article briefly recapitulates pharmacokinetic processes involved in the pulmonary drug delivery route, mechanisms of drug deposition in the respiratory tract, types of inhalable microparticles, and production methodology. An overview of recent advancements in inhalable microparticles reported in publications and patents published in the last few years has been summarized. Recent innovations in inhaler technology and major challenges in pulmonary drug delivery have been discussed briefly.

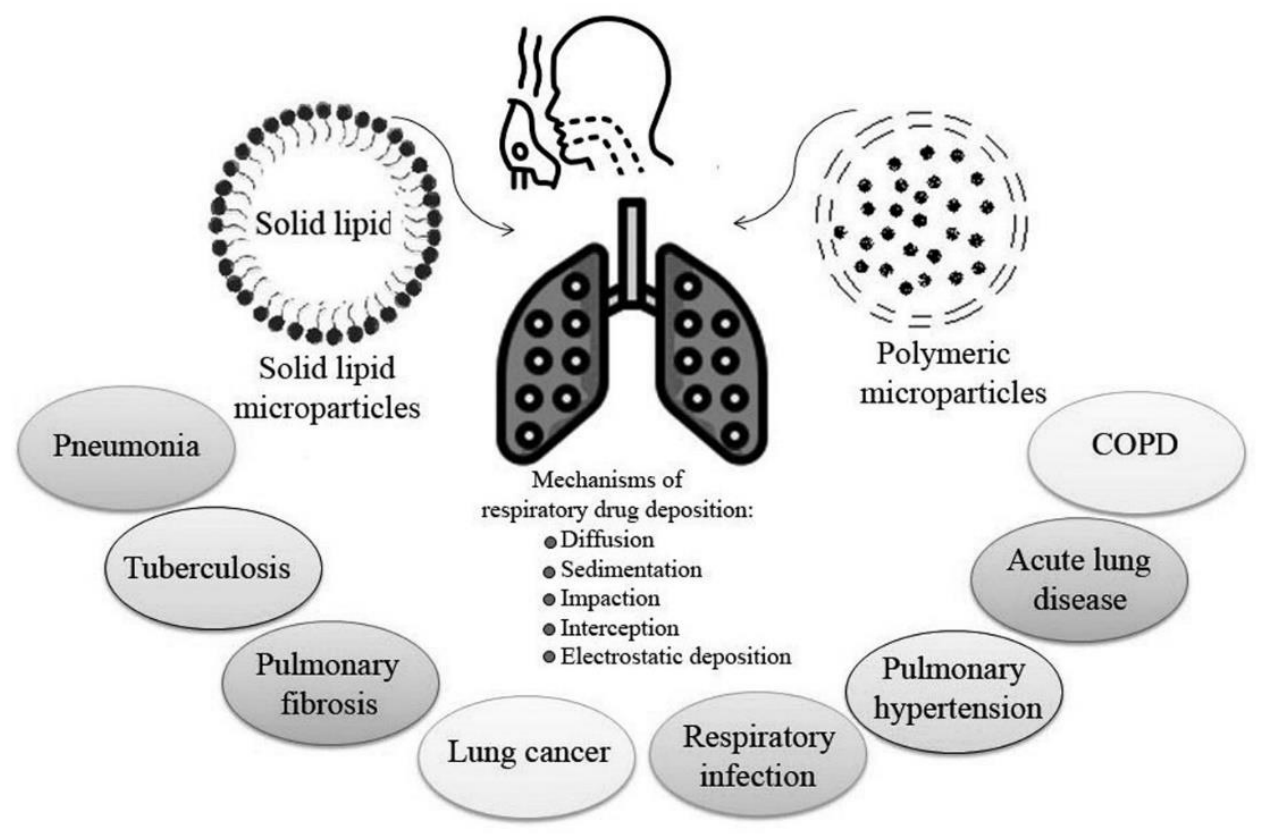

Figure 1. Application of microparticles based on pulmonary drug delivery systems for the management of various types of respiratory disorders.

\section{Pharmacokinetic Processes in Pulmonary Drug Delivery Route}

Conventional methods of administration, like intravenous and oral routes, have distinctive pharmacokinetic considerations. Before absorption into the systemic circulation, drugs taken orally shall permeate the gastrointestinal (GI) tract, portal vein, and liver. As a result, the dissolution and solubility process in GI fluid plays an important role in the rate and 
extent of absorption. Following dissolution, passive permeability, the intestinal metabolism, as well as active uptake process, regulates drug absorption through the intestinal membrane, and eventually, the drug undergoes systemic bioavailability [7,9]. Conversely, intravenous administration includes infusion or injection of drug molecules instantly into the bloodstream, thus circumventing the gastrointestinal absorption step as well as the intestinal and hepatic first metabolism [10]. However, in respiratory diseases, the main target organ is the lung. Therefore, the main drawback of oral or intravenous administration is that the target is often un-located in the plasma or blood, and consequently, pulmonary selectivity could not be achieved via systemic administration [11]. Figure 2 illustrates the six-stage pharmacokinetic processes involved subsequent to pulmonary administration.

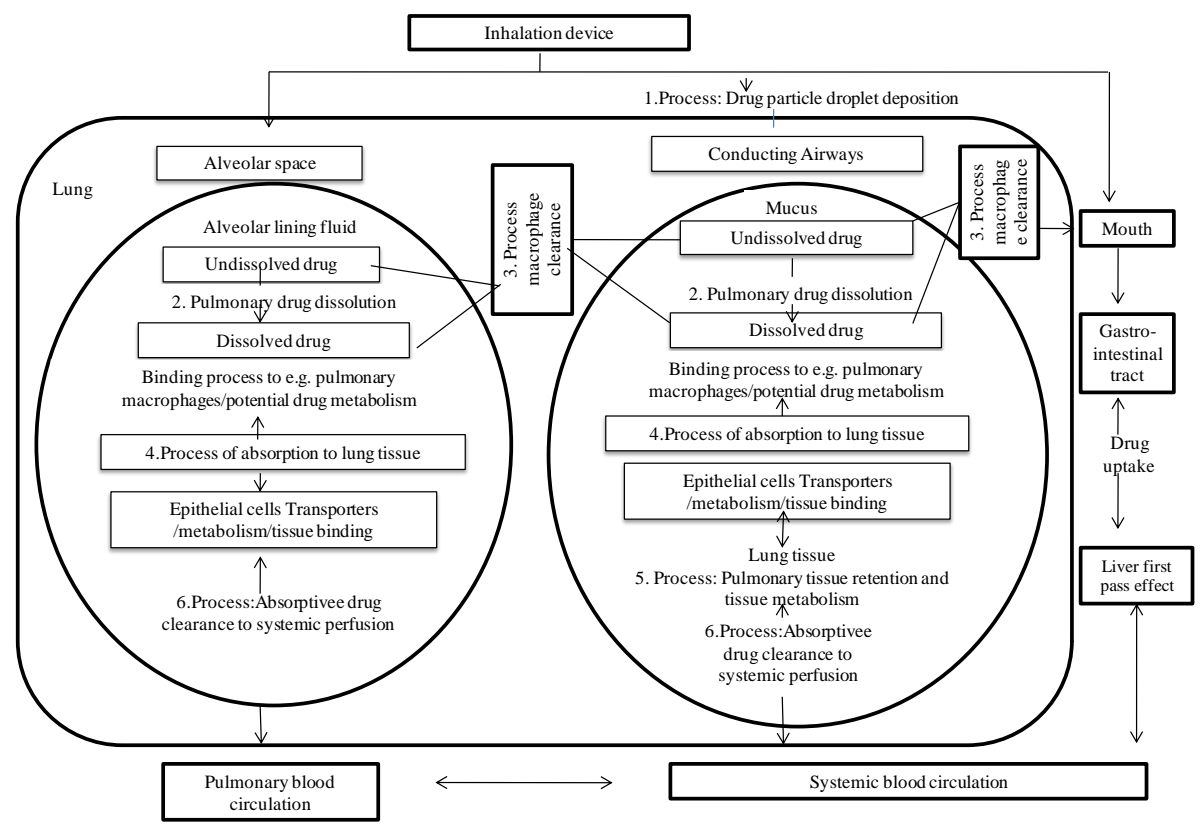

Figure 2. Pharmacokinetic processes following pulmonary drug delivery.

\subsection{Stage 1: Drug particle or droplet deposition.}

Accumulation of drug particles or droplets has been the first stage after inhalation. A small proportion of the dose gets reposed in the system, whereas the remaining drug particles or droplets get distributed to the pulmonary tract. The deposition takes place in the mouth, throat region, the conducting airways, and/or the space in the alveoli as particles travel across the airway system. The total portion of the drug accumulated in the lung is usually referred to as the lung dose. Aerodynamic diameter, inhalation flow, device properties, and disease-related factors are some of the elements that influence the dose of lung excepting the physicochemical properties of the drug itself [12-15]. Particles or droplets that fail to enter the lung are consequently swallowed, while others that enter the lung are exhaled [16,17]. Novel inhalation systems have been developed to release particles having aerodynamic diameters of $1-5 \mu \mathrm{m}$ for optimizing the lung dose and amount of particles that are effectively delivered to their target site [18].

\subsection{Stage 2: Pulmonary drug dissolution.}

Following drug particle or droplet deposition, particles must disperse within the fluids of epithelial cells. Physiologic factors, formulation of a drug as well as its physicochemical properties such as dissolution characteristics, play an essential role in pulmonary drug 
dissolution [17]. Slow dissolution as a rate-limiting step is obligatory as it prolongs lung retention, although with a simultaneous increase in the possibility that drug particles may get cleaned by mucociliary clearance.

\subsection{Stage 3: Mucociliary clearance and macrophage clearance.}

Deposited pulmonary dose, as well as lungs-specific clearance mechanisms, are a few critical factors that play a major role in deposited drug particle pulmonary bioavailability. The removal of drug molecules has been primarily achieved by mucociliary clearance, an essential mechanism of pulmonary defense throughout the respiratory tract against tiny particles or microbes [23]. Through striking the intrinsic cilia via the pharynx, an upwards passage of mucus has been carried out during this period. As a result, fine particles brought to the pharynx through mucociliary clearance have been ingested as well as distributed into the gastrointestinal tract. This phenomenon is most rapid in large air passages since mucociliary clearance improves with quite a larger airway circumference and a higher density mucus membrane [24]. The relevance of mucociliary clearance is quite complicated, which gets influenced through both medications as well as the character traits of an individual.

\subsection{Stage 4: Absorption to lung tissue.}

Active ingredients which adequately evade mechanisms of respiratory clearance as well as distribute throughout the fluid of epithelial cells might also promptly be absorbed into lung tissue. Accumulation via respiratory barriers is affected by either the features of patient-specific air passages or the characteristics of drugs. Lipophilic drugs have been easily absorbed by passive transcellular diffusion with epithelial cells upon dissolution [25]. Therefore, hydrophilic substances get absorbed through intercellular gap junctions via paracellular diffusion through the aqueous epithelium pores [26-30].

\subsection{Stage 5: Pulmonary tissue retention and tissue metabolism.}

The retention, as well as the distribution of absorbed drug throughout the pulmonary tissue, depends on the physicochemical characteristics of an inhaled drug or on patient-specific features of air passages. Amongst the most significant factors are tissue affinity or the pulmonary tissue diffusion coefficient [17,31-35].

\subsection{Stage 6: Absorptive drug clearance to the systemic perfusion.}

Pulmonary or absorptive clearance is the elimination through perfusion of the drug from the lung tissues into the bloodstream. Nevertheless, the rates of local perfusion vary within the different lung structures as well as in the alveolar area [36]. High perfusion rate facilitates quick equilibrium with alveoli systemic circulation tends to result in quite a short half-life of drug distribution in the pulmonary region. Increased respiratory specificity \& low perfusion levels combined with high tissue retention throughout the tracheobronchial location ensures maximum local airway specificity as well as the prolonged-time of equilibrium [37,38].

\section{Drug Deposition Mechanisms in Respiratory Tract}

Particle deposition mechanisms in the respiratory tract can be divided into two major categories; major mechanisms (diffusion, sedimentation, and impaction) and minor 
mechanisms (interception and electrostatic), as depicted in Figure 3 [39,40]. The aerodynamic diameter of a particle with density $1 \mathrm{gcm}^{-3}$ settles in stationary air with constant velocity [41]. Particles having aerodynamic diameters greater than $10,0.5-5$, and 2 to $0.05 \mu \mathrm{m}$ have an impact on walls of the throat, respiratory bronchioles, and alveoli, respectively [12,42-46].

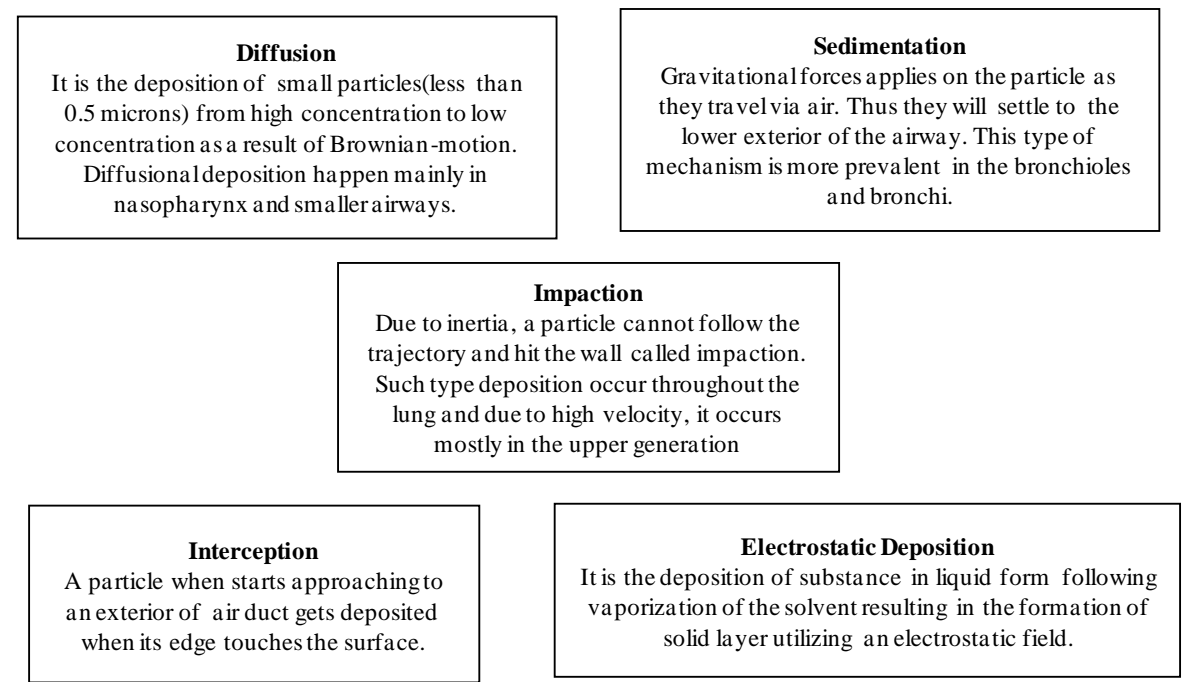

Figure 3. Mechanisms of respiratory drug deposition.

\section{Types of Inhalable Microparticles}

Microparticles-based drug delivery systems have emerged as the most significant approach for the treatment of respiratory disorders owing to improved drug's therapeutic index, prolonged biological half-life, and reduced toxicity [47]. They are micron-size particles wherein the drug is physically and uniformly dispersed enclosed by walls of synthetic and natural polymer films of varying thickness and degree of permeability acting as a release rate controlling substance. The polymeric microparticles, solid lipid microparticles, large porous microparticles, and drug-cyclodextrin complex microparticles could be utilized as inhalable microparticles [48]. In view of the fact that micro-particles do not conglomerate under shear force and deposit in the deep lung; therefore, they are gaining importance in the area of pulmonary drug delivery [49]. Inhalable microparticles utilize dry powder inhalers for drug delivery, which offers numerous advantages such as propellant-free, convenient to use, and handle. Fine particle fraction (FPF) and aerodynamic diameter are two important parameters that determine dry powder inhalers performance. Particles having an aerodynamic diameter greater than $1 \mu \mathrm{m}$ are very fine and can be simply exhaled, whereas particles having an aerodynamic diameter less than $5 \mu \mathrm{m}$ deposit in the region of upper airways. Therefore, to attain safe and effective drug delivery deep into the lungs, these must-have an aerodynamic diameter of $1-5 \mu \mathrm{m}$

\subsection{Polymeric microparticles.}

Polymeric microparticles are particulate dispersions or solid particles with size ranging from 1-1000 um. They are naturally or synthetically derived polymer and are biocompatible and biodegradable such as polylactic acid, polybutylcyanoacrylate, poly (lactic acid-co-lysine) graft, chitosan, and poly lactic-glycolic acid (PLGA) [51-53]. Wang et al. conducted in-vitro and in-vivo studies on docetaxel-loaded chitosan microspheres and found enhanced bioavailability and sustained release with minimum systemic toxicity [54]. 


\subsection{Large porous microparticles (LPMs).}

LPMs have an important role to play in the enhancement of deep lung deposition and circumventing uptake of macrophages. LPMs can be defined as light particles possessing low density $\left(<0.4 \mathrm{gcm}^{-3}\right)$ and appropriate aerodynamic diameter $(1-3 \mu \mathrm{m})$. They are extremely porous, which makes them sufficient light to reach profoundly inside the lungs and simply apprehended by lung's macrophages through inhalation. Osmogens or porogen like pluronic, cyclodextrins, hydrogen peroxide, or catalase (gas bubbles) and gas-foaming (ammonium bicarbonate) are utilized for the production of LPMs. Both osmogens and porogens undergo loss out of drugs by surface pores, as a consequence of water channels made inside polymeric matrix [55-58].

\subsection{Solid lipid microparticles (SLMs).}

SLMs have spherical shapes and sizes in the range of 1 to $1000 \mu \mathrm{m}$. They are lipid matrix composed of glyceride, fatty acid, fatty alcohol, and solid wax, possessing high melting points. In recent years, SLMs could serve as a good alternative and acceptable method for pulmonary drug delivery. Currently, for the development of inhalable SLMs, cisplatin was mixed with a solubilized lipid and PEGylated component using high-pressure homogenization and spray-dried $[59,60]$.

\subsection{Cyclodextrin complex microparticles.}

Cyclodextrins (CDs) like $\alpha-, \beta$ - and $\gamma$-CDs is composed of cyclic oligosaccharides that are cyclic polymers of $\alpha$-D-glycopyranose. Because of their high complexation efficiency, high loading capacity along with the low cost of production, $\beta$-CDs are frequently used for pharmaceutical applications. Due to their exceptional structure, having an external layer comprising hydroxyl groups along with the lipophilic internal cavity, they result in inclusion complexation formation with the hydrophobic drugs. To reduce the drug irritation following pulmonary delivery CDs have proven to be excellent solubilizing agents in aerosol preparations of poorly water-insoluble drugs. Therefore, CDs are broadly applied as drug carriers for the pulmonary route of drug administration. A safe $\beta$-CD derivative, a novel inhalable dry powder formulation of Fisetin (an inclusion complex with sulfobutyl ether- $\beta$-cyclodextrin), was developed to augment the aqueous solubility of a drug intended to be used for pulmonary drug delivery $[60,61]$.

\section{Production Methodology of Inhalable Microparticles}

\subsection{Emulsion polymerization technique.}

Emulsion polymerization is a simple process in which the monomers are stabilized by using surfactants and are dispersed in an aqueous phase. The obtained microparticles display a low polydispersity index and produce high yields [62].

\subsection{Dry coating technique.}

In this technique, the prepared microparticles are lyophilized and introduced into Mechanofusion $^{\mathrm{TM}}$ apparatus chamber simultaneously with lactose (Pharmatose $325 \mathrm{M}^{\mathrm{TM}}$ ). This apparatus consists of a rotating chamber having 200-1600 rpm speed, a stationary blade, and a 
scrapper. Microparticles are adsorbed onto the surface of lactose, which can be easily deposited inside the lung [63] (Figure 4).

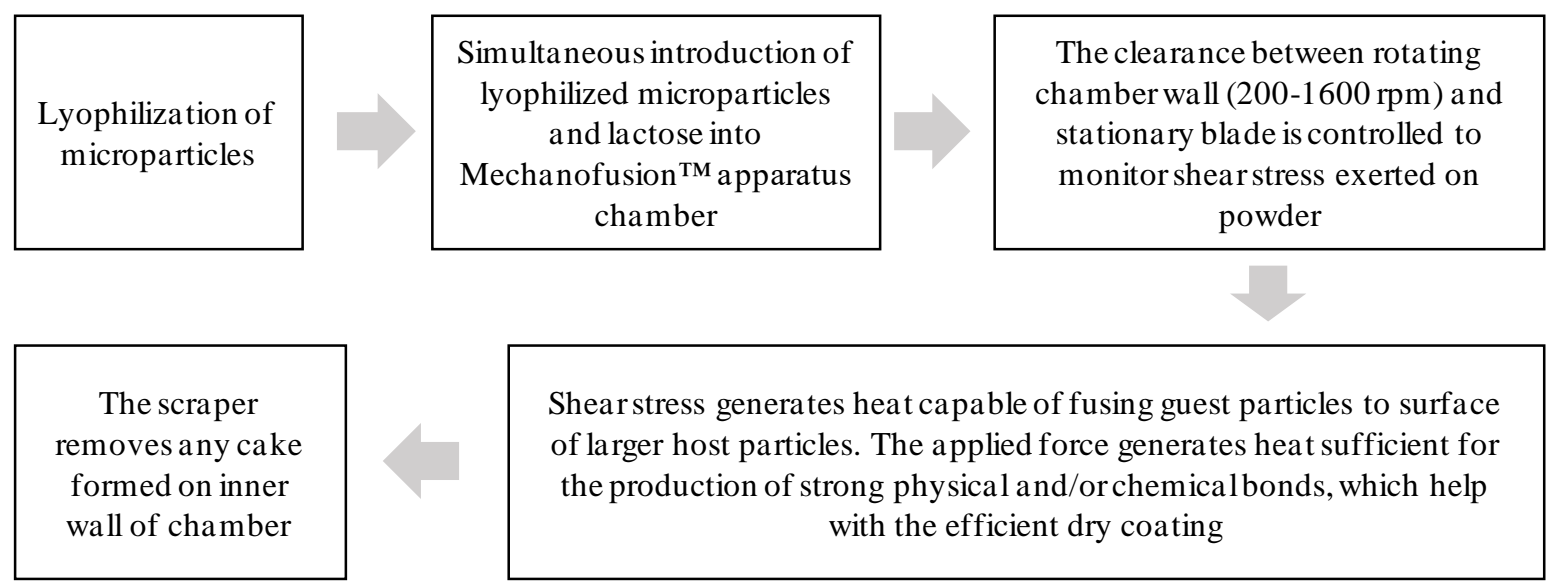

Figure 4. Dry coating technique for microparticles production.

\subsection{Air suspension technique.}

The process is also known as the Wurster process, was designed by Dale E. Wurster in 1940. Wurster coating is the process of encapsulation of distinct particles by means of differential airflow to create a cyclic movement of material in a fluidized bed (Figure 5) [64].

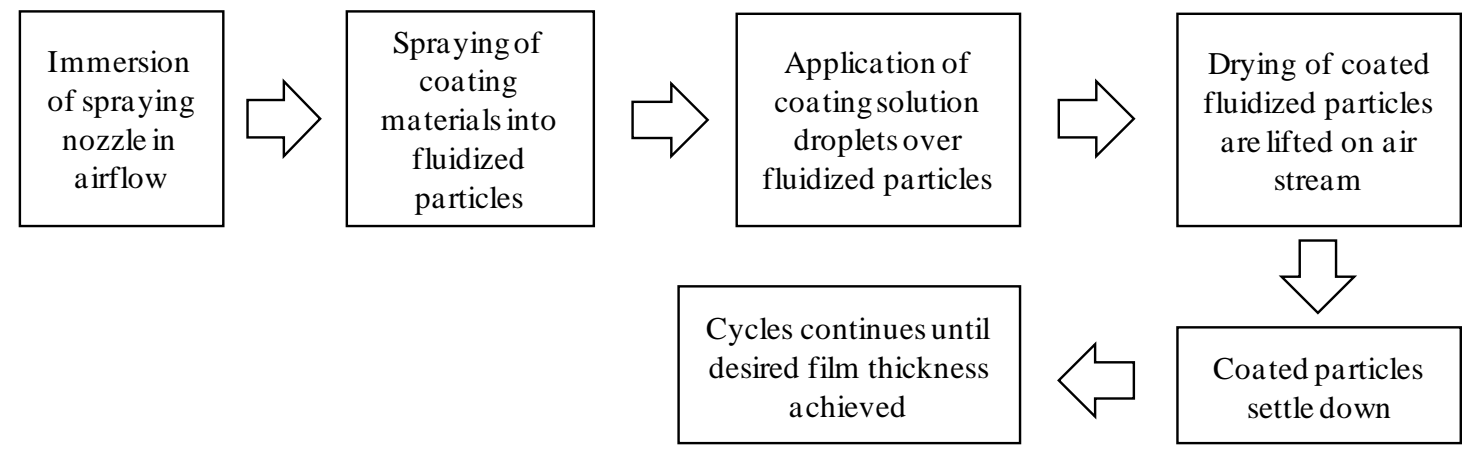

Figure 5. Air suspension technique for microparticles production.

\subsection{Spray drying technique.}

This technique was a breakthrough in the pharmaceutical industry over previous technologies. It is a dehydration method having high speed, versatility, high encapsulation efficiencies, and easy to scale-up. Parameters that influence the size and morphology of the microparticles are inlet-outlet temperature, nozzle diameter, air or solution volume mixture, pressure, feed rate, and type of atomizer [65] (Figure 6).

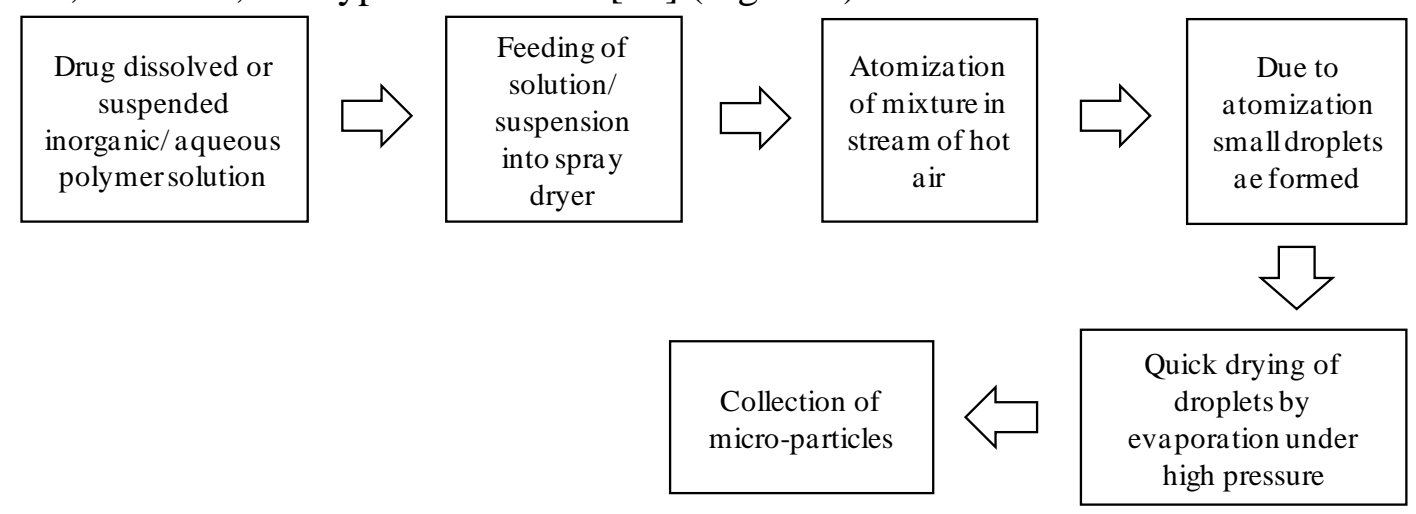

Figure 6. Spray drying technique for microparticles production. 


\subsection{Spray freeze-drying method.}

Spray-freeze-drying is appropriate for extremely thermo-sensitive materials and particularly for lipid microparticles that are unable to withstand the high temperature during the predictable drying process, even for a short time. In this technique, the microparticles are protected from different stresses such as freezing and dehydration through immobilization in the glassy matrix of polysaccharide (Figure 7). However, a crystallization inhibitor is used to maintain the microparticles and polysaccharide in an amorphous state [65].

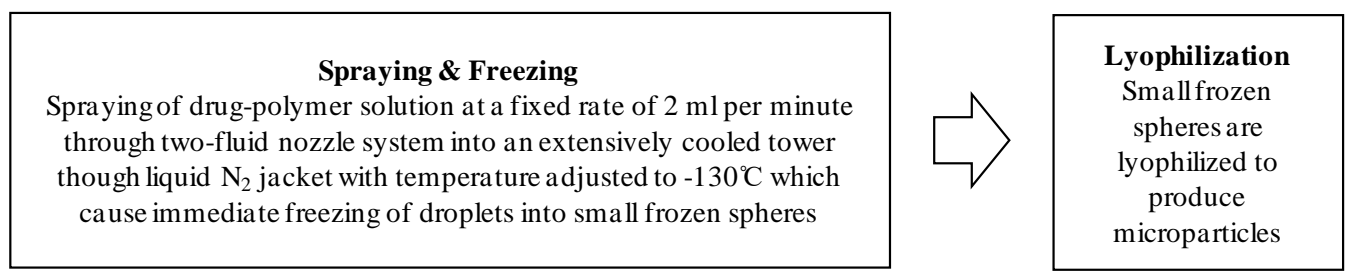

Figure 7. Spray freeze-drying technique for microparticles production.

\section{Recent Advancements in Inhalable Microparticles}

In view of the fact that lungs are in continuous contact with air, they are susceptible to numerous disorders and diseases like respiratory infections, asthma, chronic obstructive pulmonary disease, pneumonia, tuberculosis, acute respiratory distress syndrome in infants, and chronic lung cancers [3]. Therefore, studies had been conducted to explore the potential of microparticles in pulmonary drug delivery. Kim et al. developed inhalable sustained-release microparticles for co-delivery of doxorubicin along with TRAIL (type II transmembrane protein that stimulates programmed cell death in numerous carcinoma cells) for treatment of metastatic lung carcinoma. Furthermore, the interaction between doxorubicin and Trail suggests that the doxorubicin dosage and its side effects could be significantly reduced [66]. In another study, Zhu and its colleagues evaluated the effect of LPMs for in-situ treatment of primary non-small lung carcinoma. The authors conclude that oridonin plus PLGA-based LPPs possess a geometric diameter of about $\sim 10 \mu \mathrm{m}$ and an aerodynamic diameter of about $2.72 \mu \mathrm{m}$ leading to very effective lung deposition [67]. Furthermore, Nafee and its colleagues prepared inhalable microparticles incorporating myricetin SLNs for lung carcinoma. It was established that optimized SLNs have a diameter of $75.98 \mathrm{~nm}$, a zeta-potential of $-22.5 \mathrm{mV}$, and an encapsulation efficiency of $84.5 \%$. In addition, it was concluded that phospholipid complexation provides a five-fold improvement in the loading of drugs [68]. In research performed by Reczyńskaa and its colleagues, paclitaxel loaded microparticles were combined with superparamagnetic iron oxide nanoparticles. In-vitro efficacies against malicious epithelial cells of lungs (A549) of microparticles were assessed and found inhibited colony formation and substantially decreased cell viability [69]. In another study, Chaurasiya et al. fabricated paclitaxel loaded bovine serum albumin microparticles as dry powders having dimensions in the range of 0.5 to $3.0 \mu \mathrm{m}$. A comparison between formulated microparticles and commercially available taxol compound was made. It was observed that in comparison with taxol, the anticancer activity of developed microparticles showed better drug release profiles [70]. Furthermore, Tewes and colleagues formulated porous microparticles comprising superparamagnetic iron oxide nanoparticles supported by a target-directed magnetic inclined field for cancer nodules [71]. In addition, Cunha and his colleagues prepared an inhalable formulation of chitosan microparticles of rifabutin and isoniazid for lung tuberculosis therapy [72]. It was found that chitosan microparticles are possessing large encapsulation efficiencies 
(93\% for isoniazid and $99 \%$ for rifabutin) along with aerodynamic diameter $(4 \mu \mathrm{m})$. In another study, Omar et al. formulated isoniazid-loaded chitosan microparticles and concluded that minimum inhibitory concentration of microparticles showed 63 -fold more inhibition on Mycobacterium tuberculosis than isoniazid solution, due to the synergism of positively charged microparticles with their cellular penetration activity and promoting their bacterial cell surface binding [73]. In another study, $\mathrm{Ni}$ and its colleagues designed microparticles embedded in nanocrystals for sustained pulmonary drug delivery of cinaciguat. It was further established that microparticles have a mean volume diameter of about 3-4 $\mu \mathrm{m}$, an aerodynamic diameter of about 4-4.5 $\mu \mathrm{m}, \mathrm{FPF}$ of 40-45\%, and 94-95\% emitted dose [74]. In another study, Dufour et al. developed CDs-based spray-dried microparticles incorporating budesonide for the management of bronchial inflammation associated with asthma. It was found FPF was increased from $26.24 \%$ (with lactose) to $44.05 \%$ (with CDs) [75]. In another study conducted by Mahmoud and a group of researchers, microparticles of alginate were formulated for the pulmonary delivery of roflumilast. It was further reported that microparticles utilizing CDs efficaciously suppress the viability of pro-inflammatory cytokines (TNF- $\alpha$, IL-6, and IL-10) in comparison with pure drugs [76]. In a recent study, Hu et al. developed inhalable curcumin incorporated PLGA-LPMs and concluded that curcumin LPMs showed potential effects in the treatment of idiopathic pulmonary fibrosis [77]. Jaspart and its colleagues formulated SLMs of salbutamol acetonide. It was found that in comparison with the pure drug, the drug release can be sustained by SLMs [78]. Furthermore, another team of researchers developed microparticles containing clarithromycin for inhalation using chitosan and leucine. It was found that clarithromycin microparticles are capable of interfering with bacteria proliferation, therefore, making it appropriate for pulmonary drug delivery [79]. Another study involved the formulation of inhalable silk-based microparticles to deliver high-payload ciprofloxacin for non-cystic fibrosis bronchiectasis. It was found that the optimal formulation exhibited emitted dose $(98.10 \pm 1.27 \%)$, geometric standard deviation (1.66 \pm 0.10$)$, good aerosol efficiency with regard to FPF $(45.04 \pm 0.84 \%)$, and aerodynamic diameter $(3.75 \pm 0.03 \mu \mathrm{m})$ along with the highest drug loading of $80 \%$ [80]. Polymeric microparticles of sodium cromoglycate using carboxymethylcellulose, sodium alginate, and sodium hyaluronate were fabricated by the spray-drying method. Drug release, in-vitro aerosolization, and mucoadhesion properties were studied and concluded that polymeric microparticles of sodium cromoglycate promise to be an attractive mucoadhesive inhalable formulation [81].

Table 1. Literature reports on inhalable microparticles for the treatment of respiratory disorders.

\begin{tabular}{l|l|l|l|l|l} 
Model Drug & Polymers & Type of microparticles & Methodology & Application & Ref. \\
\hline Paclitaxel & Sodium alginate & $\begin{array}{l}\text { Polymeric } \\
\text { microparticles }\end{array}$ & $\begin{array}{l}\text { Emulsification } \\
\text { technique }\end{array}$ & Lung cancer & {$[49]$} \\
\hline Doxorubicin & PLGA & $\begin{array}{l}\text { Sustained-release } \\
\text { polymeric } \\
\text { microparticles }\end{array}$ & $\begin{array}{l}\text { w/o/w double } \\
\text { emulsification }\end{array}$ & Lung cancer & {$[66]$} \\
\hline Oridonin & PLGA & $\begin{array}{l}\text { Large porous } \\
\text { microparticles }\end{array}$ & $\begin{array}{l}\text { Solvent } \\
\text { evaporation/ } \\
\text { emulsion/freeze- } \\
\text { drying method }\end{array}$ & $\begin{array}{l}\text { Non-small cell } \\
\text { lung carcinoma }\end{array}$ & {$[67]$} \\
\hline $\begin{array}{l}\text { Myricetin } \\
\text { (flavonoid) }\end{array}$ & $\begin{array}{l}\text { Lipoid@-S100, } \\
\text { Gelucire-based } \\
\text { surfactant, Mannitol, } \\
\text { Maltodextrin, L- } \\
\text { leucine }\end{array}$ & $\begin{array}{l}\text { SLN loaded respirable } \\
\text { microparticles }\end{array}$ & $\begin{array}{l}\text { Complexation/ } \\
\text { nano- } \\
\text { encapsulation/ } \\
\text { Spray drying }\end{array}$ & Lung cancer & [68] \\
\hline $\begin{array}{l}\text { Iron } \\
\text { Paclitaxel }\end{array}$ & $\begin{array}{l}\text { Myristic, palmitic, } \\
\text { Lauric acid }\end{array}$ & $\begin{array}{l}\text { Nanoparticles- } \\
\text { ombedded } \\
\text { microparticles }\end{array}$ & $\begin{array}{l}\text { Hot oil-in-water } \\
\text { emulsification }\end{array}$ & Lung cancer & [69] \\
\end{tabular}




\begin{tabular}{|c|c|c|c|c|c|}
\hline Model Drug & Polymers & Type of microparticles & Methodology & Application & Ref. \\
\hline Paclitaxel & $\begin{array}{ll}\begin{array}{l}\text { Bovine } \\
\text { albumin }\end{array} & \text { serum } \\
\end{array}$ & SLM & $\begin{array}{ll}\text { Spray drying } \\
\text { technique }\end{array}$ & $\begin{array}{l}\text { Metastatic Lung } \\
\text { Cancer }\end{array}$ & [70] \\
\hline $\begin{array}{l}\text { Super } \\
\text { paramagnetic } \\
\text { iron oxide } \\
\text { microparticles } \\
\end{array}$ & $\begin{array}{l}\text { 2-hydroxy-propyl- } \beta \text { - } \\
\text { cyclodextrin, } \\
\text { Polyethylene glycol }\end{array}$ & Trojan microparticles & Spray drying & $\begin{array}{l}\text { Specific } \quad \text { lung } \\
\text { delivery under a } \\
\text { magnetic gradient } \\
\text { field }\end{array}$ & [71] \\
\hline $\begin{array}{l}\text { Isoniazid/ } \\
\text { Rifabutin }\end{array}$ & Chitosan & SLM & Spray drying & $\begin{array}{l}\text { Pulmonary } \\
\text { tuberculosis }\end{array}$ & [72] \\
\hline Isoniazid & Chitosan & SLM & Spray drying & Tuberculosis & [73] \\
\hline Cinaciguat & Chitosan & $\begin{array}{l}\text { Polymeric } \\
\text { microparticles }\end{array}$ & $\begin{array}{l}\text { High-pressure } \\
\text { homogenization }\end{array}$ & $\begin{array}{l}\text { Pulmonary } \\
\text { hypertension }\end{array}$ & [74] \\
\hline Budesonide & $\begin{array}{l}\text { Hydroxypropyl- } \beta- \\
\text { cyclodextrin }\end{array}$ & $\begin{array}{l}\text { Polymeric } \\
\text { microparticles }\end{array}$ & Spray drying & Asthma & {$[75]$} \\
\hline Roflumilast & $\begin{array}{l}\text { Sodium alginate, } \\
\text { Calcium beta-glycero } \\
\text { phosphate }\end{array}$ & SLM & Spray drying & $\begin{array}{l}\text { Tumor-associated } \\
\text { inflammation }\end{array}$ & [76] \\
\hline Curcumin & PLGA & Porous microparticles & $\begin{array}{l}\text { Emulsion } \\
\text { solvent } \\
\text { evaporation }\end{array}$ & $\begin{array}{l}\text { Idiopathic } \\
\text { Pulmonary } \\
\text { Fibrosis }\end{array}$ & [77] \\
\hline $\begin{array}{l}\text { Salbutamol } \\
\text { acetonide }\end{array}$ & Glyceryl behenate & SLM & $\begin{array}{l}\text { Hot emulsion } \\
\text { high-shear } \\
\text { homogenization }\end{array}$ & $\begin{array}{l}\text { Asthma and } \\
\text { COPD }\end{array}$ & [78] \\
\hline Clarithromycin & Leucine and Chitosan & SLM & $\begin{array}{l}\text { Controlled } \\
\text { magnetic stirring }\end{array}$ & Tuberculosis & [79] \\
\hline Ciprofloxacin & Mannitol, Silk fibroin & $\begin{array}{l}\text { Silk-based } \\
\text { microparticles }\end{array}$ & Spray drying & $\begin{array}{l}\text { Non-cystic } \\
\text { fibrosis } \\
\text { bronchiectasis }\end{array}$ & [80] \\
\hline $\begin{array}{l}\text { Sodium } \\
\text { Cromoglycate }\end{array}$ & $\begin{array}{l}\text { Sodium hyaluronate, } \\
\text { Sodium alginate, } \\
\text { Sodium CMC } \\
\end{array}$ & $\begin{array}{l}\text { Polymeric } \\
\text { microparticles }\end{array}$ & Spray drying & Asthma & [81] \\
\hline $\begin{array}{l}\text { Isoniazid and } \\
\text { Rifabutin }\end{array}$ & Poly (1-lactic acid) & $\begin{array}{l}\text { Dry powder inhalable } \\
\text { polymeric } \\
\text { microparticles }\end{array}$ & Spray drying & Tuberculosis & {$[82]$} \\
\hline $\begin{array}{l}\text { Di-sodium } \\
\text { cromoglycate }\end{array}$ & PVA & $\begin{array}{l}\text { Controlled release co- } \\
\text { spray dried polymeric } \\
\text { microparticles }\end{array}$ & Spray drying & $\begin{array}{l}\text { Asthma and } \\
\text { respiratory tract } \\
\text { inflammation }\end{array}$ & [83] \\
\hline Budesonide & $\begin{array}{ll}\text { Pluronic } & \text { F-68, } \\
\text { Compritol } 888 & \end{array}$ & SLM & $\begin{array}{l}\text { Phase inversion } \\
\mathrm{o} / \mathrm{w} \text { emulsion } \\
\text { technique and } \\
\text { spray drying }\end{array}$ & Asthma & [84] \\
\hline Budesonide & Chitosan & $\begin{array}{l}\text { Polymeric } \\
\text { microparticles }\end{array}$ & Spray drying & Asthma & [85] \\
\hline Thymopentin & Mannitol, Leucine & SLM & Co-spray drying & $\begin{array}{l}\text { Immuno- } \\
\text { modulator in } \\
\text { autoimmune } \\
\text { diseases, } \\
\text { infections and } \\
\text { cancers }\end{array}$ & {$[86]$} \\
\hline Cyclosporin & Mannitol & $\begin{array}{l}\text { Polymeric } \\
\text { microparticles }\end{array}$ & $\begin{array}{ll}\begin{array}{l}\text { Spray } \\
\text { drying }\end{array} & \text { freeze- } \\
\end{array}$ & COPD & [87] \\
\hline Rifampicin & $\begin{array}{l}\text { Chitosan, ascorbic } \\
\text { acid, Leucine }\end{array}$ & $\begin{array}{l}\text { Polymeric } \\
\text { microparticles }\end{array}$ & Spray drying & Tuberculosis & [88] \\
\hline $\begin{array}{l}\text { Doxycycline } \\
\text { hyclate }\end{array}$ & $\begin{array}{l}\text { Leucine, Lactose, } \\
\text { Sodium alginate, } \\
\text { PVA PVP, Starch } \\
\end{array}$ & $\begin{array}{l}\text { Mucoadhesive } \\
\text { polymeric } \\
\text { microparticles }\end{array}$ & Spray drying & Tuberculosis & [89] \\
\hline Heparin & $\begin{array}{lr}\text { PLGA; } & \text { Resomer® } \\
\text { grades } & \text { RG502, } \\
\text { RG503, } & \text { RG504 } \\
\end{array}$ & $\begin{array}{l}\text { Extended-release } \\
\text { polymeric } \\
\text { microparticles }\end{array}$ & Spray drying & $\begin{array}{l}\text { Airway hyper- } \\
\text { reactivity and } \\
\text { inflammation }\end{array}$ & [90] \\
\hline Quercetin & $\begin{array}{l}\text { Tristearin, } \\
\text { Phosphatidylcholine }\end{array}$ & SLM & $\begin{array}{l}\text { Oil in water } \\
\text { emulsification by } \\
\text { phase inversion } \\
\text { with Spray drying }\end{array}$ & COPD & [91] \\
\hline Dexamethasone & $\begin{array}{l}\text { Poly-caprolactoone, } \\
\text { Sodium } \\
\text { deoxycholate }\end{array}$ & $\begin{array}{l}\text { Polymeric } \\
\text { microparticles }\end{array}$ & $\begin{array}{l}\text { Vibrational Spray } \\
\text { drying }\end{array}$ & Asthma & [92] \\
\hline $\begin{array}{l}\text { Salbutamol } \\
\text { sulfate }\end{array}$ & $\begin{array}{l}\text { Dipalmitoyl- } \\
\text { phosphatidyl- }\end{array}$ & SLM & Spray drying & $\begin{array}{l}\text { Asthma } \\
\text { COPD }\end{array}$ & [93] \\
\hline
\end{tabular}




\begin{tabular}{|c|c|c|c|c|c|}
\hline Model Drug & Polymers & Type of microparticles & Methodology & Application & Ref. \\
\hline & $\begin{array}{l}\text { choline, Cholesterol, } \\
\text { L-leucine }\end{array}$ & & & & \\
\hline Sildenafil & $\begin{array}{ll}\text { PLGA, } & \text { Resomer® } \\
\text { RG502H } & \\
\end{array}$ & $\begin{array}{l}\text { Polymeric } \\
\text { microparticles }\end{array}$ & Spray drying & $\begin{array}{l}\text { Respiratory tract } \\
\text { infections }\end{array}$ & [94] \\
\hline Resveratrol & $\begin{array}{l}\text { Tristearin, Chitosan, } \\
\text { Phosphatidyl-choline }\end{array}$ & SLM & $\begin{array}{l}\text { Melt } \\
\text { emulsification }\end{array}$ & $\begin{array}{l}\text { Nasal } \\
\text { Inflammation }\end{array}$ & [95] \\
\hline $\begin{array}{ll}\text { Isoniazid } & \text { or } \\
\text { Rifabutin } & \\
\end{array}$ & Locust bean gum & $\begin{array}{l}\text { Polymeric } \\
\text { microparticles }\end{array}$ & Spray drying & Tuberculosis & [96] \\
\hline Dexamethasone & $\begin{array}{l}\text { Vanillyl alcohol-with } \\
\text { co-polyoxalate }\end{array}$ & Porous microparticles & $\begin{array}{l}\text { Double } \\
\text { emulsification }\end{array}$ & $\begin{array}{l}\text { Airway } \\
\text { inflammatory } \\
\text { disease } \\
\end{array}$ & {$[97]$} \\
\hline Insulin & $\begin{array}{ll}\text { Chitosan, } & \text { Methyl } \\
\text { iodide, } & \text { Sodium } \\
\text { iodide } & \end{array}$ & $\begin{array}{l}\text { Polymeric } \\
\text { microparticles }\end{array}$ & $\begin{array}{l}\text { Supercritical fluid } \\
\text { assisted } \\
\text { atomization with } \\
\text { hydro-dynamic } \\
\text { cavitation mixer }\end{array}$ & Diabetes mellitus & [98] \\
\hline Azithromycin & $\begin{array}{l}\text { Fumaryl } \\
\text { diketopiperazine }\end{array}$ & $\begin{array}{l}\text { Respirable } \\
\text { microparticles }\end{array}$ & Spray drying & Pneumonia & [99] \\
\hline Rifampicin & $\begin{array}{l}\text { L-leucine, } \\
\text { L-aspartic acid }\end{array}$ & $\begin{array}{l}\text { Polymeric } \\
\text { microparticles }\end{array}$ & $\begin{array}{l}\text { Spray dryer via } \\
\text { emulsion }\end{array}$ & Tuberculosis & [100] \\
\hline Indomethacin & $\begin{array}{l}\text { Epsilon-polylysine, } \\
\text { Dextrin, Lactose } \\
\text { monohydrate }\end{array}$ & $\begin{array}{l}\text { Dry powder inhalable } \\
\text { microparticles }\end{array}$ & Spray drying & $\begin{array}{l}\text { Bronchial } \\
\text { inflammatory } \\
\text { asthma } \\
\end{array}$ & [101] \\
\hline $\begin{array}{l}\text { Salmeterol } \\
\text { Xinafoate }\end{array}$ & $\begin{array}{lr}\text { Pluronic } & \text { F-68, } \\
\text { DDAB, } & \text { Sodium } \\
\text { alginate, Mucin }\end{array}$ & SLM & Freeze Drying & COPD & [102] \\
\hline Sildenafil citrate & $\begin{array}{l}\text { Sodium alginate, } \\
\text { Sodium CMC, } \\
\text { Sodium hyaluronate, } \\
\text { L-leucine }\end{array}$ & Hydrogel microparticles & Spray drying & $\begin{array}{l}\text { Pulmonary } \\
\text { hypertension }\end{array}$ & [103] \\
\hline Budenoside & PLGA, PVP & $\begin{array}{ll}\text { Large } & \text { porous } \\
\text { microparticles } & \end{array}$ & $\begin{array}{ll}\text { Modified } & \mathrm{o} / \mathrm{w} \\
\text { solvent } & \\
\text { evaporation } & \\
\end{array}$ & Asthma & [104] \\
\hline $\begin{array}{l}\text { Trans-Retinoic } \\
\text { acid }\end{array}$ & PLGA & Spray drying & Spray-drying & Tuberculosis & {$[105]$} \\
\hline $\begin{array}{l}\text { Isoniazid/ } \\
\text { Rifabutin }\end{array}$ & $\begin{array}{l}\text { Konjac glucomanna } \\
\mathrm{n}\end{array}$ & $\begin{array}{l}\text { Polymeric } \\
\text { microparticles }\end{array}$ & Spray-drying & Tuberculosis & [106] \\
\hline $\begin{array}{l}\text { Chloramphenicol } \\
\text { palmitate/ } \\
\text { Thiamphenicol } \\
\text { palmitate }\end{array}$ & $\begin{array}{l}\text { PLGA, Lactose } \\
\text { monohydrate, } \\
\text { Leucine }\end{array}$ & $\begin{array}{l}\text { Sustained-release nano- } \\
\text { embedded } \\
\text { microparticles }\end{array}$ & $\begin{array}{l}\text { Emulsion solvent } \\
\text { evaporation with } \\
\text { spray-drying }\end{array}$ & $\begin{array}{l}\text { Multidrug- } \\
\text { resistant } \\
\text { pulmonary } \\
\text { bacterial } \\
\text { infections } \\
\end{array}$ & [107] \\
\hline Ciprofloxacin & Chitosan & $\begin{array}{l}\text { Polymeric } \\
\text { microparticles }\end{array}$ & Ionic gelation & $\begin{array}{l}\text { Antibacterial } \\
\text { activity in lungs }\end{array}$ & [108] \\
\hline
\end{tabular}

Abbreviations: COPD: Chronic obstructive pulmonary disease; DDAB: dimethyldioctadecyl-ammonium bromide; DOTAP: Dioleoyltrimethylammoniumpropane; Fumaryl diketopiperazine: Synthesized via three-step reaction using $\mathrm{N}_{6}$-trifluoro-acetyl-L-lysine, phosphorus pent-oxide, $\mathrm{N}$-methyl-2-pyrrolidinone; Sodium CMC: Sodium carboxymethyl cellulose; PLGA: Poly (D, L-Lactide-co-glycolide); PVA: Polyvinyl alcohol; PVP: Polyvinyl pyrollidone, Resomer® RG 502H: PLGA 50:50 (MW: 7000-17,000, acid terminated); SLM: Solid lipid microparticles.

Recent publications of inhalable microparticles for pulmonary administration are mentioned in Table 1. Recently published patents of inhalable microparticles have been summarized in Table 2.

Table 2. Recent patents of inhalable microparticles.

\begin{tabular}{l|l|l|l} 
Patent No. & Invention & Date of Publication & Ref. \\
\hline US005451569A & Pulmonary drug delivery system & 19 September 1995 & {$[109]$} \\
\hline US005855913A & $\begin{array}{l}\text { Particles incorporating surfactants for pulmonary drug } \\
\text { delivery, unit dose micro-cartridges dry powder, } \\
\text { related devices, and methods }\end{array}$ & January 1999 & {$[110]$} \\
\hline WO1996036314A3 & Method for drug delivery to the pulmonary system & 21 November 1996 & {$[111]$} \\
\hline WO2004030659A1 & Sustained-release porous microparticles for inhalation & 15 April 2004 & {$[112]$} \\
\hline US20050084455 & $\begin{array}{l}\text { Inhalable biodegradable microparticles for target- } \\
\text { specific drug delivery in tuberculosis and a process } \\
\text { thereof }\end{array}$ & 21 April 2005 & {$[113]$}
\end{tabular}




\begin{tabular}{l|l|l|l}
\hline Patent No. & Invention & Date of Publication & Ref. \\
\hline US2007154408 & $\begin{array}{l}\text { Inhalable Biodegradable microparticles for target- } \\
\text { specific drug delivery in tuberculosis and a process } \\
\text { thereof. }\end{array}$ & 5uly 2007 & {$[114]$} \\
\hline US 20070215149A1 & Dry powder inhalers having spiral travel paths & 20 September 2007 & {$[115]$} \\
\hline US7625865B2 & Insulin highly respirable microparticles & 1 December 2009 & {$[116]$} \\
\hline WO2012017405A1 & $\begin{array}{l}\text { Microparticles formulation for pulmonary drug } \\
\text { delivery of anti-infective molecule for the treatment of } \\
\text { infectious diseases }\end{array}$ & 9 February 2012 & {$[117]$} \\
\hline US8227409B2 & Diketopiperazine with defined isomer contents & 24 July 2012 & {$[118]$} \\
\hline US00916 1901 B2 & $\begin{array}{l}\text { Nanoparticles Targeted drug delivery to the lungs using } \\
\text { extra-testicular Sertoli cells }\end{array}$ & 20 October 2015 & {$[119]$} \\
\hline WO 2016/019253 Al & Dry powder formulations for inhalation & 4 February 2016 & {$[120]$} \\
\hline JP2016222689 & Compositions for treating pain & 28 December 2016 & {$[121]$} \\
\hline US10342938B2 & Dry powder drug delivery system & 9 July 2019 & {$[122]$}
\end{tabular}

\section{Recent Innovations in Inhaler Technology}

The pulmonary route has been employed to cure a variety of respiratory diseases from older times. Numerous ancient inhalation therapies that are used to suppress cough are vapors from aromatic herbs or plants, balsams, myrrh, and leaves from plants. Adrenaline had been discovered as a nebulizer solution in the 1920s; in 1925, porcine insulin has been used as a nebulizer for diabetes mellitus in experimental studies, and in 1945 newly discovered penicillin was tested for pulmonary delivery $[123,124]$. The modern inhalation devices have been listed in Figure 8. Dry powder inhalers (DPIs) are usually employed for the alleviation of respiratory disorders, namely bronchitis, emphysema, and chronic obstructive pulmonary disease (COPD). DPI had also been used for treating diabetes mellitus as inhalable insulin. Recent innovations in inhaler technology of marketed inhalers are described in Table 3.
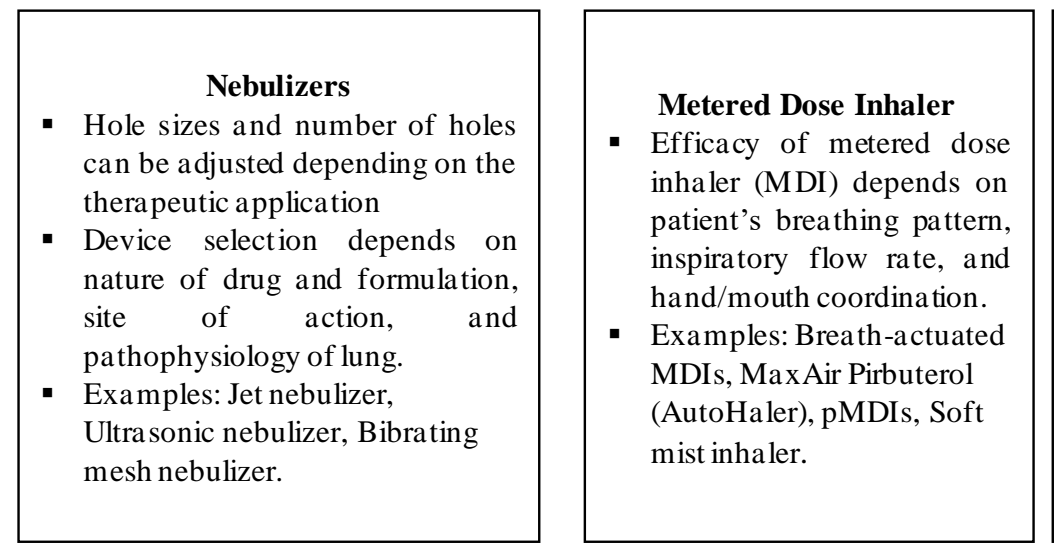

\section{Dry Powder Inhaler}

- Important parameters are effect of airflow changes and deagglomeration in inhaler device.

- Examples: Spinhaler (Fisons Pharmaceuticals, Rochester), Rotahaler (GSK, RTP)

Figure 8. Types and examples of pulmonary drug delivery devices.

Table 3. Recent innovations in inhaler technology of marketed inhalers.

\begin{tabular}{|c|c|c|c|}
\hline $\begin{array}{l}\text { Marketed } \\
\text { Brand }\end{array}$ & Manufacturer & Innovation & Ref. \\
\hline $\begin{array}{l}\text { Technosphere }{ }^{\circledR} \\
\text { Insulin }\end{array}$ & Mankind & $\begin{array}{l}\text { It includes a dry-powder composition of insulin in crystal form filled in } \\
\text { gelatin capsules. They show very fast absorption as investigated in } \\
\text { pharmacokinetics as well as pharmacodynamics studies. }\end{array}$ & [125] \\
\hline Exubera ${ }^{\circledR}$ & Nektar/ Pfizer & $\begin{array}{l}\text { It uses insulin powder preparation, consisting of excipients like glycine, } \\
\text { mannitol, along with } 60 \text { percent recombinant human insulin. } \\
\text { Pharmacokinetics of insulin using inhalation therapy showed a peak } \\
\text { round about } 55 \text { min and quick return in comparison with subcutaneous } \\
\text { insulin. }\end{array}$ & [126] \\
\hline Aerodose ${ }^{\circledR}$ & $\begin{array}{ll}\text { Aerogen } & \text { Inc./ } \\
\text { Nektar } & \\
\end{array}$ & $\begin{array}{l}\text { It is a system using insulin in liquid form aerosolized in the shape of } \\
\text { small droplets. }\end{array}$ & [127] \\
\hline Spiro system & $\begin{array}{l}\text { Dina Pharmacy } \\
\text { Inc/Elan } \\
\text { Corporation }\end{array}$ & $\begin{array}{l}\text { It offers a dry-powder insulin preparation coated in blister-disks through } \\
\text { a breath-actuated inhaler. }\end{array}$ & [128] \\
\hline
\end{tabular}




\begin{tabular}{l|l|l|l}
$\begin{array}{l}\text { Marketed } \\
\text { Brand }\end{array}$ & Manufacturer & Innovation & Ref. \\
\hline $\begin{array}{l}\text { DCI } \\
\text { Dose counter } \\
\text { inhalers) }\end{array}$ & Dr. Reddy's & $\begin{array}{l}\text { It is a new drug delivery device with a single device having 120 metered } \\
\text { doses. There is a window in the inhaler that changes color from green to } \\
\text { red. }\end{array}$ & {$[129]$} \\
\hline Turbohaler & Astra Zeneca & $\begin{array}{l}\text { This is a 'breath-activated' system, which means that dry powder } \\
\text { medicine gets sucked from the system rather than being discharged, as } \\
\text { those from other devices. }\end{array}$ & $\begin{array}{l}\text { [130] } \\
\text { delivers approximately 91-112 percent of metered dose }\end{array}$ \\
\hline Twisthaler & $\begin{array}{l}\text { Schering/ } \\
\text { Mercks }\end{array}$ & $\begin{array}{l}\text { This is an eco-friendly, reliable, and convenient to be used for asthma } \\
\text { and COPD therapy. }\end{array}$ & {$[131]$} \\
\hline Easyhaler & Orion & Help in delivering morphine and insulin into the lungs. & {$[131]$} \\
\hline AERx & Aradigm & & $133]$
\end{tabular}

\section{Major Challenges in Pulmonary Drug Delivery}

\subsection{Low-performance inhalation system.}

The primary standoff confronting pulmonary drug delivery is often the low efficiency of the inhalation systems available today. The optimum particle size of the aerosol is needed to deliver drugs more deeply into the lung. The optimum particle size for deep lung deposition is 1-5 $\mu \mathrm{m}$. The pharmaceutical aerosol system must produce particles of desired fine size particles. If the particles are of larger size, they may affect larynx and oropharynx [3].

\subsection{Less drug mass per puff and poor formulation stability.}

Delivery of many drugs requires milligram doses needed for pulmonary delivery, but mostly in current systems, the total drug per puff carried to the lower respiratory tract is much lower than $1000 \mu \mathrm{g}$. The stability of asthmatic drugs is more in liquids in comparison to dry state [3].

\subsection{Inappropriate dosing reproducibility.}

A problem in delivery device and instability of formulation are some of the reasons responsible for improper dosing reproducibility. Therefore, patient education is required to get maximum dose reproducibility [3].

\section{Conclusions}

Nowadays, pulmonary drug delivery is becoming very relevant because of large absorptive surface area, elimination of the first-pass metabolism, and very thin diffusion layer that enables rapid absorption of drugs at the target site. Presently, the key drug targeting systems involve drug delivery straightforwardly into the lungs, primarily using inhalation treatment via dry powder inhalers and pressurized metered-dose inhalers. Therefore, inhalable microparticles having aerodynamic diameters of $1-5 \mu \mathrm{m}$ could be successfully and effectively explored in forthcoming years for the management of respiratory diseases such as cystic fibrosis, COPD, pneumonia, chronic bronchitis, tuberculosis, lung cancer, and asthma.

\section{Current \& Future Developments}

Due to the broad absorptive surface region, elimination of the first-pass metabolism, and quite a thin diffusion layer that allows for quick drug absorption at the targeted lung cells, pulmonary drug delivery is nowadays gaining considerable importance. Polymeric 
microparticles, large porous microparticles, solid lipid microparticles, and cyclodextrin complex microparticles having aerodynamic diameters of 1-5 $\mu \mathrm{m}$ could be successfully and effectively explored as inhalable microparticles for producing superior therapeutic effect along with minimized systemic toxicity for respiratory diseases management in forthcoming years.

\section{Funding}

This research received no external funding.

\section{Acknowledgments}

The authors express gratitude to Chitkara College of Pharmacy, Chitkara University, Punjab, India, for motivational support for the compilation of this review.

\section{Conflicts of Interest}

The authors declare no conflict of interest.

\section{References}

1. Mansour, H.M.; Rhee, Y.S.; Wu, X. Nanomedicine in pulmonary delivery. Int. J. Nanomedicine 2009, 4, 299-319, https://doi.org/10.2147/ijn.s4937.

2. Azarmi, S.; Tao, X.; Chen, H.; Wang, Z.; Finlay, W.H.; Löbenberg, R.; Roa, W.H. Formulation and cytotoxicity of doxorubicin nanoparticles carried by dry powder aerosol particles. Int. J. Pharm. 2006, 319, 155-161, https://doi.org/10.1016/j.ijpharm.2006.03.052.

3. Paranjpe, M.; Müller-Goymann, C.C. Nanoparticle-mediated pulmonary drug delivery: a review. Int. J. Mol. Sci. 2014, 15, 5852-5873, https://doi.org/10.3390/ijms15045852.

4. Mehta, P.; Bothiraja, C.; Mahadik, K.; Kadam, S.; Pawar, A. Phytoconstituent based dry powder inhalers as biomedicine for the management of pulmonary diseases. Biomed. Pharmacother. 2018, 108, 828-837, https://doi.org/10.1016/j.biopha.2018.09.094.

5. Xia, Y.; Su, Y.; Wang, Q.; Yang, C.; Tang, B.; Zhang, Y.; Tu, J.; Shen, Y. Preparation, characterization, and pharmacodynamics of insulin-loaded fumaryl diketopiperazine microparticle dry powder inhalation. Drug Deliv. 2019, 26, 650-660, https://doi.org/10.1080/10717544.2019.1631408.

6. Ferkol, T.; Schraufnagel, D. The global burden of respiratory disease. Ann. Am. Thorac. Soc. 2014, 11, 404406, https://doi.org/10.1513/AnnalsATS.201311-405PS.

7. Borghardt, J.M.; Kloft, C.; Sharma, A. Inhaled therapy in respiratory disease: the complex interplay of pulmonary kinetic processes. Can. Respir. J. 2018, 2018, 1-18. https://doi.org/10.1155/2018/2732017.

8. Patil, J.S.; Sarasija, S. Pulmonary drug delivery strategies: A concise, systematic review. Lung India 2012, 29, 44-49, https://doi.org/10.4103/0970-2113.92361.

9. Zhao, Y.H.; Abraham, M.H.; Le, J.; Hersey, A.; Luscombe, C.N.; Beck, G.; Sherborne, B.; Cooper, I. Ratelimited steps of human oral absorption and QSAR studies. Pharm. Res. 2002, 19, 1446-1457, https://doi.org/10.1023/a:1020444330011.

10. Jin, J.F.; Zhu, L.L.; Chen, M.; Xu, H.M.; Wang, H.F.; Feng, X.Q.; Zhu, X.P.; Zhou, Q. The optimal choice of medication administration route regarding intravenous, intramuscular, and subcutaneous injection. Patient Prefer Adherence 2015, 9, 9-23, https://doi.org/10.2147/PPA.S87271.

11. Jones, H.M.; Rowland-Yeo, K. Basic concepts in physiologically based pharmacokinetic modeling in drug discovery and development. CPT Pharmacometrics Syst. Pharmacol. 2013, 2, 1-2, https://doi.org/10.1038/psp.2013.41.

12. Labiris, N.R.; Dolovich, M.B. Pulmonary drug delivery. Part I: physiological factors affecting therapeutic effectiveness of aerosolized medications. Br. J. Clin. Pharmacol. 2003, 56, 588-599, https://doi.org/10.1046/j.1365-2125.2003.01892.x.

13. Tsuda, A.; Henry, F.S.; Butler, J.P. Particle transport and deposition: basic physics of particle kinetics. Compr. Physiol. 2011, 3, 1437-1471, https://doi.org/10.1002/cphy.c100085.

14. Thorsson, L.; Geller, D. Factors guiding the choice of delivery device for inhaled corticosteroids in the longterm management of stable asthma and COPD: focus on budesonide. Resp. Med. 2005, 99, 836-849, https://doi.org/10.1016/j.rmed.2005.02.012.

15. Ganguly, K.; Carlander, U.; Garessus, E.D.; Fridén, M.; Eriksson, U.G.; Tehler, U.; Johanson, G. Computational modeling of lung deposition of inhaled particles in chronic obstructive pulmonary disease 
(COPD) patients: identification of gaps in knowledge and data. Crit. Rev. Toxicol. 2019, 49, 160-173, https://doi.org/10.1080/10408444.2019.1584153.

16. Chrystyn, H. Methods to identify drug deposition in the lungs following inhalation. Br. J. Clin. Pharmacol. 2001, 51, 289-299, https://doi.org/10.1046/j.1365-2125.2001.01304.x.

17. Hochhaus, G.; Horhota, S.; Hendeles, L.; Suarez. S.; Rebello, J. Pharmacokinetics of orally inhaled drug products. AAPS J. 2015, 17, 769-775, https://doi.org/10.1208/s12248-015-9736-6.

18. Borghardt, J.M.; Weber, B.; Staab, A.; Kloft, C. Pharmacometric models for characterizing the pharmacokinetics of orally inhaled drugs. The AAPS J. 2015, 17, 853-870, https://doi.org/10.1208/s12248015-9760-6.

19. Daley-Yates, P.T. Inhaled corticosteroids: potency, dose equivalence and therapeutic index. Br. J. Clin. Pharmacol. 2015, 80, 372-380, https://doi.org/10.1111/bcp.12637.

20. Johnson, M. Pharmacodynamics and pharmacokinetics of inhaled glucocorticoids. J. Allergy Clin. Immunol. 1996, 97, 169-176, https://doi.org/10.1016/S0091-6749(96)80217-X.

21. Keller, J.G.; Graham, U.M.; Koltermann-Jülly, J.; Gelein, R.; Ma-Hock, L.; Landsiedel, R.; Wiemann, M.; Oberdörster, G.; Elder, A.; Wohlleben, W. Predicting dissolution and transformation of inhaled nanoparticles in the lung using abiotic flow cells: The case of barium sulfate. Sci. Rep. 2020, 10, 1-15, https://doi.org/10.1038/s41598-019-56872-3.

22. Yalkowsk, S.; He, Y.; Jain, P. Handbook of Aqueous Solubility Data. Taylor \& Francis Inc., Oxford, UK. 2010.

23. Chae, H.S.; Kim, S.Y.; Pel, P.; Huh, J.; Joo, S.W.; Lim, Y.Y.; Park, S.J.; Lim, J.L.; Chin, Y.W. Standardized Extract of Atractylodis Rhizoma Alba and Fructus Schisandrae Ameliorates Coughing and Increases Expectoration of Phlegm. Molecules 2020, 25, 3064-3076, https://doi.org/10.3390/molecules25133064.

24. Foster, W.M.; Langenback, E.; Bergofsky, E.H. Measurement of tracheal and bronchial mucus velocities in man: relation to lung clearance. J. Appl. Physiol. 1980, 48, 965-971, https://doi.org/10.1152/jappl.1980.48.6.965.

25. Woods, A.; Andrian, T.; Sharp, G.; Bicer, E.M.; Vandera, K.K.A.; Patel, A.; Mudway, I.; Dailey, L.A.; Forbesa, B. Development of new in vitro models of lung protease activity for investigating stability of inhaled biological therapies and drug delivery systems. Eur. J. Pharm. Biopharm. 2020, 146, 64-72, https://doi.org/10.1016/j.ejpb.2019.11.005.

26. Patton, J.S.; Fishburn, C.S.; Weers, J.G. The lungs as a portal of entry for systemic drug delivery. Ann. Am. Thorac. Soc. 2004, 1 338-344.

27. Olsson, B.; Bondesso, E.; Borgstrom, L.; Edsbäcker, S.; Eirefelt, S.; Ekelund, K.; Gustavsson, L.; HegelundMyrbäck, T. Pulmonary drug metabolism, clearance, and absorption. In: Controlled Pulmonary Drug Delivery. Smyth, H.; Hickey, A. Ed.; Springer, New York, NY, USA, 2011; pp. 21-50, https://doi.org/10.1007/978-1-4419-9745-6_2.

28. Eriksson, J.; Sjögren, E.; Lennernäs, H.; Thörn, H. Drug absorption parameters obtained using the isolated perfused rat lung model are predictive of rat in vivo lung absorption. AAPS J. 2020, 22, 71-83, https://doi.org/10.1208/s12248-020-00456-x.

29. Ruge, C.A.; Kirch, J.; Lehr, C.M. Pulmonary drug delivery: from generating aerosols to overcoming biological barriers-therapeutic possibilities and technological challenges. Lancet Respir. Med. 2013, 1, 402413, https://doi.org/10.1016/S2213-2600(13)70072-9.

30. Movia, D.; Prina-Mello, A. Preclinical development of orally inhaled drugs (oids)-are animal models predictive or shall we move towards in vitro non-animal models? Animals (Basel) 2020, 10, 1259-1275, https://doi.org/10.3390/ani10081259.

31. Derendorf, H. Excessive lysosomal ion-trapping of hydroxychloroquine and azithromycin. Int. J. Antimicrob. Agents 2020, 55, 106007-106013, https://doi.org/10.1016/j.ijantimicag.2020.106007.

32. Borghardt, J.M.; Weber, B.; Staab, A.; Kunz, C.; Formella, S.; Kloft, C. Investigating pulmonary and systemic pharmacokinetics of inhaled olodaterol in healthy volunteers using a population pharmacokinetic approach. Br. J. Clic. Pharmacol. 2011, 81, 538-552, https://doi.org/10.1111/bcp.12780.

33. Bartels, C.; Looby, M.; Sechaud, R.; Kaiser, G. Determination of the pharmacokinetics of glycopyrronium in the lung using a population pharmacokinetic modelling approach. Br. J. Clin. Pharmacol. 2013, 76, 868879, https://doi.org/10.1111/bcp.12118.

34. Weber, B.; Troconiz, I.F.; Borghardt, J.M.; Staab, A.; Sharma, A. Model-based evaluation of single and multiple dose pharmacokinetics of inhaled tiotropium in healthy volunteers and implications for systemic exposure studies. Respiratory Drug Del. Europe 2015, 2, 249-254.

35. Edsbäcker, S.; Johansson, C.J. Airway selectivity: an update of pharmacokinetic factors affecting local and systemic disposition of inhaled steroids. Basic Clin. Pharmacol. 2006, 98, 523-536, https://doi.org/10.1111/j.1742-7843.2006.pto_355.x.

36. Lin, Z.; Li, M.; Wang, Y.S.; Tell, L.A.; Baynes, R.E.; Davis, J.L.; Vickroy, T.W.; Riviere, J.E. Physiological parameter values for physiologically based pharmacokinetic models in food-producing animals. Part I: Cattle and swine. J. Vet. Pharmacol. Ther. 2020, 43, 385-420, https://doi.org/10.1111/jvp.12861.

37. Boger, E.; Evans, N.; Chappell, M.; Lundqvist, A.; Ewing, P.; Wigenborg, A.; Fridén, M. Systems pharmacology approach for prediction of pulmonary and systemic pharmacokinetics and receptor occupancy 
of inhaled drugs. CPT Pharmacometrics Syst. Pharmacol. 2016, 5, 201-210, https://doi.org/10.1002/psp4.12074.

38. Bäckström, E.; Hamm, G.; Nilsson, A.; Fihn, B.M.; Strittmatter, N.; Andrén, P.; Goodwin, R.J.; Fridén, M. Uncovering the regional localization of inhaled salmeterol retention in the lung. Drug Deliv. 2018, 25, 838845, https://doi.org/10.1080/10717544.2018.1455762.

39. Ashish, K.; Hiralal, C.; Prajkata, U.; Dheeraj, B.; Dinesh, K. Pulmonary drug delivery system. Int. J. Pharm.Tech. Res. 2012, 4, 293-305.

40. Kadam, P.; Kanekar, H.; Khale, A. Pulmonary drug delivery system: current practices and applications. World J. Pharm. Res. 2014, 3, 204-229.

41. Shaikh, S.; Nazim, S.; Khan, T.; Shaikh, A.; Zameeruddin, M.; Quazi, A. Recent advances in pulmonary drug delivery system: A review. Int. J. Appl. Pharm. 2010, 2, 27-33.

42. Cheng, Y.S. Mechanisms of pharmaceutical aerosol deposition in the respiratory tract. AAPS PharmSciTech. 2014, 15, 630-640, https://doi.org/10.1208/s12249-014-0092-0.

43. Groneberg, D.A.; Witt, C.; Wagner, U.; Chung, K.F.; Fischer, A. Fundamentals of pulmonary drug delivery. Respir. Med. 2003, 97, 382-387, https://doi.org/10.1053/rmed.2002.1457.

44. Thulasiramaraju, T.V.; Kumar, B.T.; Babu, M.N. Pulmonary drug delivery system: An overview. AJRBPS 2013, $1,16-34$

45. Shah, N.D.; Shah, V.V.; Chivate, N. Pulmonary drug delivery: a promising approach. J. Appl. Pharm. Sci. 2012, 2, 33-37.

46. Ibrahim, M.; Verma, R.; Contreras, L.G. Inhalation drug delivery devices: technology update. Med Devices (Auckl) 2015, 8, 131-139, https://doi.org/10.2147/MDER.S48888.

47. Alipour, S.; Montaseri, H.; Tafaghodi, M. Preparation and characterization of biodegradable paclitaxel loaded alginate microparticles for pulmonary delivery. Colloid Surface B. 2010, 81, 521-529, https://doi.org/10.1016/j.colsurfb.2010.07.050.

48. El-Sherbiny, I.M.; El-Baz, N.M.; Yacoub, M.H. Inhaled nano-and microparticles for drug delivery. Glob. Cardiol. Sci. Pract. 2015, 2015, 1-14, https://doi.org/10.5339/gcsp.2015.2.

49. Abdelaziz, H.M.; Gaber, M.; Abd-Elwakil, M.M.; Mabrouk, M.T.; Elgohary, M.M.; Kamel, N.M.; Kabary, D.M.; Freag, M.S.; Samaha, M.W.; Mortada, S.M.; Elkhodairy, K.A. Inhalable particulate drug delivery systems for lung cancer therapy: Nanoparticles, microparticles, nanocomposites and nanoaggregates. $J$. Control Release 2018, 269, 374-392, https://doi.org/10.1016/j.jconrel.2017.11.036.

50. Goel, A.; Baboota, S.; Sahni, J.K.; Ali, J. Exploring targeted pulmonary delivery for treatment of lung cancer. Int. J. Pharm. Investig. 2013, 3, https://doi.org/10.4103/2230-973X.108959.

51. Lee, W.H.; Loo, C.Y.; Traini, D.; Young, P.M. Inhalation of nanoparticle-based drug for lung cancer treatment: advantages and challenges. Asian J. Pharm. Sci. 2015, 10, 481-489, https://doi.org/10.1016/j.ajps.2015.08.009.

52. Hitzman, C.J.; Elmquist, W.F.; Wattenberg, L.W.; Wiedmann, T.S. Development of a respirable, sustained release microcarrier for 5-fluorouracil I: In vitro assessment of liposomes, microspheres, and lipid coated nanoparticles. J. Pharm. Sci. 2006, 95, 1114-1126, https://doi.org/10.1002/jps.20591.

53. Williams III, R.O.; Barron, M.K.; Alonso, M.J.; Remuñán-López, C. Investigation of a pMDI system containing chitosan microspheres and P134a. Int. J. Pharm. 1998, 174, 209-222, https://doi.org/10.1016/S0378-5173(98)00266-X.

54. Wang, H.; Xu, Y.; Zhou, X. Docetaxel-loaded chitosan microspheres as a lung targeted drug delivery system: in vitro and in vivo evaluation. Int. J. Mol. Sci. 2014, 15, 3519-3532, https://doi.org/10.3390/ijms15033519.

55. Kim, I.; Byeon, H.J.; Kim, T.H.; Lee, E.S.; Oh, K.T.; Shin, B.S.; Lee, K.C.; Youn, Y.S. Doxorubicin-loaded highly porous large PLGA microparticles as a sustained-release inhalation system for the treatment of $\begin{array}{lllll}\text { metastatic lung } & \text { cancer. } & \text { Biomaterials }\end{array}$ https://doi.org/10.1016/j.biomaterials.2012.04.018.

56. Wu, D.; Wang, C.; Yang, J.; Wang, H.; Han, H.; Zhang, A.; Yang, Y.; Li, Q. Improving the intracellular drug concentration in lung cancer treatment through the co-delivery of doxorubicin and miR-519c mediated by porous PLGA microparticle. Mol. Pharm. 2016, 13, 3925-3933, https://doi.org/10.1021/acs.molpharmaceut.6b00702.

57. Zhu, L.; Li, M.; Liu.; X.; Du, L.; Jin, Y. Inhalable oridonin-loaded poly (lactic-co-glycolic) acid large porous microparticles for in situ treatment of primary non-small cell lung cancer. Acta Pharm. Sin. B 2017, 7, 8090, https://doi.org/10.1016/j.apsb.2016.09.006.

58. Umeyor, C.E.; Kenechukwu, F.C.; Uronnachi, E.M.; Osonwa, U.E.; Nwakile, C.D. Solid lipid microparticles (SLMs): an effective lipid based technology for controlled drug delivery. Am. J. Pharmtech Res. 2012, 2, $1-18$.

59. Levet, V.; Rosière, R.; Merlos, R.; Fusaro, L.; Berger, G.; Amighi, K.; Wauthoz, N. Development of controlled-release cisplatin dry powders for inhalation against lung cancers. Int. J. Pharm. 2016, 515, 209220, https://doi.org/10.1016/j.ijpharm.2016.10.019.

60. Gidwani, B.; Vyas, A. A comprehensive review on cyclodextrin-based carriers for delivery of chemotherapeutic cytotoxic anticancer drugs. Biomed. Res. Int. 2015, 2015, 1-15, https://doi.org/10.1155/2015/198268. 
61. Mohtar, N.; Taylor, K.M.; Sheikh, K.; Somavarapu, S. Design and development of dry powder sulfobutylether- $\beta$-cyclodextrin complex for pulmonary delivery of fisetin. Eur. J. Pharm. Biopharm. 2017, 113, 1-10, https://doi.org/10.1016/j.ejpb.2016.11.036.

62. Kumar, S.; Nakka, S.; Rajabalaya, R.; Kumar, H.; Halder, T.; Palanisamy, M.; Nanda, A. Microencapsulation techniques and its practices. Int. J. Pharm. Sci. Techno. 2011, 6, 1-23.

63. Ezhilarasi, P.N.; Karthik, P.; Chhanwal, N.; Anandharamakrishnan, C. Nanoencapsulation techniques for food bioactive components: a review. Food Bioproc. Tech. 2013, 6, 628-647, https://doi.org/10.1007/s11947-012-0944-0.

64. Wurster, D.E.; Bhattacharjya, S.; Flanagan, D.R. Effect of curing on water diffusivities in acrylate free films as measured via a sorption technique. AAPS J. 2007, 8, 152-157, https://doi.org/10.1208/pt0803071.

65. Emami, F.; Vatanara, A.; Park, E.J.; Na, D.H. Drying technologies for the stability and bioavailability of biopharmaceuticals. Pharmaceutics 2018, 10, https://doi.org/10.3390/pharmaceutics10030131.

66. Kim, I.; Byeon, H.J.; Kim, T.H.; Lee, E.S.; Oh, K.T.; Shin, B.S.; Lee, K.C.; Youn, Y.S. Doxorubicin-loaded porous PLGA microparticles with surface attached TRAIL for the inhalation treatment of metastatic lung cancer. Biomaterials 2013, 34, 6444-6453, https://doi.org/10.1016/j.biomaterials.2013.05.018.

67. Zhu, L.; Li, M.; Liu, X.; Du, L.; Jin, Y. Inhalable oridonin-loaded poly (lactic-co-glycolic) acid large porous microparticles for in situ treatment of primary non-small cell lung cancer. Acta Pharm. Sin. B 2017, 7, 8090, https://doi.org/10.1016/j.apsb.2016.09.006.

68. Nafee, N.; Gaber, D.M.; Elzoghby, A.O.; Helmy, M.W.; Abdallah, O.Y. Promoted antitumor activity of myricetin against lung carcinoma via nanoencapsulated phospholipid complex in respirable microparticles. Pharm. Res. 2020, 37, 1-24, https://doi.org/10.1007/s11095-020-02794-z.

69. Reczyńska, K.; Marszałek, M.; Zarzycki, A.; Reczyński, W.; Kornaus, K.; Pamuła, E.; Chrzanowski, W. Superparamagnetic iron oxide nanoparticles modified with silica layers as potential agents for lung cancer treatment. J. Nanomater. 2020, 10, https://doi.org/10.3390/nano10061076.

70. Chaurasiya, B.; Huang, L.; Du, Y.; Tang, B.; Qiu, Z.; Zhou, L.; Tu, J.; Sun, C. Size-based anti-tumoral effect of paclitaxel loaded albumin microparticle dry powders for inhalation to treat metastatic lung cancer in a mouse model. Int. J. Pharm. 2018, 542, 90-99, https://doi.org/10.1016/j.ijpharm.2018.02.042.

71. Tewes, F.; Ehrhardt, C.; Healy, A.M. Superparamagnetic iron oxide nanoparticles (SPIONs)-loaded Trojan microparticles for targeted aerosol delivery to the lung. Eur. J. Pharm. Biopharm. 2014, 86, 98-104, https://doi.org/10.1016/j.ejpb.2013.09.004.

72. Cunha, L.; Rodrigues, S.; Rosa da Costa, A.M.; Faleiro, L.; Buttini, F.; Grenha, A. Inhalable chitosan microparticles for simultaneous delivery of isoniazid and rifabutin in lung tuberculosis treatment. Drug Dev. Ind. Pharm. 2019, 45, 1313-1320, https://doi.org/10.1080/03639045.2019.1608231.

73. Omar, S.M.; Maziad, N.A.; El-Tantawy, N.M. Pulmonary delivery of isoniazid in nanogel-loaded chitosan hybrid microparticles for inhalation. J. Aerosol Med. Pulm. Drug Deliv. 2019, 32, 78-87, https://doi.org/10.1089/jamp.2018.1460.

74. Ni, R.; Zhao, J.; Liu, Q.; Liang, Z.; Muenster, U.; Mao, S. Nanocrystals embedded in chitosan-based respirable swellable microparticles as dry powder for sustained pulmonary drug delivery. Eur. J. Pharm. Sci. 2017, 99, 137-146, https://doi.org/10.1016/j.ejps.2016.12.013.

75. Dufour, G.; Bigazzi, W.; Wong, N.; Boschini, F.; De Tullio, P.; Piel, G.; Cataldo, D.; Evrard, B. Interest of cyclodextrins in spray-dried microparticles formulation for sustained pulmonary delivery of budesonide. Int. J. Pharm. 2015, 495, 869-878, https://doi.org/10.1016/j.ijpharm.2015.09.052.

76. Mahmoud, A.A.; Elkasabgy, N.A.; Abdelkhalek, A.A. Design and characterization of emulsified spray dried alginate microparticles as a carrier for the dually acting drug roflumilast. Eur. J. Pharm. Sci. 2018, 122, 6476, https://doi.org/10.1016/j.ejps.2018.06.015.

77. Hu, Y.; Li, M.; Zhang, M.; Jin, Y. Inhalation treatment of idiopathic pulmonary fibrosis with curcumin large porous microparticles. Int. J. Pharm. 2018, 551, 212-222, https://doi.org/10.1016/j.ijpharm.2018.09.031.

78. Jaspart, S.; Bertholet, P.; Piel, G.; Dogné, J.M.; Delattre, L.; Evrard, B. Solid lipid microparticles as a sustained release system for pulmonary drug delivery. Eur. J. Pharm. Biopharm. 2007, 65, 47-56, https://doi.org/10.1016/j.ejpb.2006.07.006.

79. Dimer, F.; de Souza Carvalho-Wodarz, C.; Haupenthal, J.; Hartmann, R.; Lehr, C.M. Inhalable clarithromycin microparticles for treatment of respiratory infections. Pharm. Res. 2015, 32, 3850-3861, https://doi.org/10.1007/s11095-015-1745-8.

80. Liu, C.; Lin, L.; Huang, Z.; Wu, Q.; Jiang, J.; Lv, L.; Yu, X.; Quan, G.; Li, G.; Wu, C. Novel inhalable ciprofloxacin dry powders for bronchiectasis therapy: mannitol-silk fibroin binary microparticles with highpayload and improved aerosolized properties. AAPS PharmaSciTech J. 2019, 20, https://doi.org/10.1208/s12249-019-1291-5.

81. Gallo, L.; Bucalá, V.; Ramírez-Rigo, M.V. Formulation and characterization of polysaccharide microparticles for pulmonary delivery of sodium cromoglycate. AAPS PharmaSciTech J. 2017, 18, 16341645, https://doi.org/10.1208/s12249-016-0633-9.

82. Muttil, P.; Kaur, J.; Kumar, K.; Yadav, A.B.; Sharma, R.; Misra, A. Inhalable microparticles containing large payload of anti-tuberculosis drugs. Eur. J. Pharm. Sci. 2007, 32, 140-150, https://doi.org/10.1016/j.ejps.2007.06.006. 
83. Salama, R.; Hoe, S.; Chan, H.K.; Traini, D.; Young, P.M. Preparation and characterization of controlled release co-spray dried drug-polymer microparticles for inhalation 1: Influence of polymer concentration on physical and in vitro characteristics. Eur. J. Pharm. Biopharm. 2008, 69, 486-495, https://doi.org/10.1016/j.ejpb.2007.12.019.

84. Mezzena, M.; Scalia, S.; Young, P.M.; Traini, D. Solid lipid budesonide microparticles for controlled release inhalation therapy. AAPS J. 2009, 11, 771-778, https://doi.org/10.1208/s12248-009-9148-6.

85. Naikwade, S.R.; Bajaj, A.N.; Gurav, P.; Gatne, M.M.; Soni, P.S. Development of budesonide microparticles using spray-drying technology for pulmonary administration: design, characterization, in vitro evaluation, and in vivo efficacy study. AAPS Pharm. Sci. Tech. 2009, 10, 993-1012, https://doi.org/10.1208/s12249009-9290-6.

86. Li, Y.Z.; Sun, X.; Gong, T.; Liu, J.; Zuo, J.; Zhang, Z.R. Inhalable microparticles as carriers for pulmonary delivery of thymopentin-loaded solid lipid nanoparticles. Pharm. Res. 2010, 27, 1977-1986, https://doi.org/10.1007/s11095-010-0201-z.

87. Niwa, T.; Mizutani, D.; Danjo, K. Spray freeze-dried porous microparticles of a poorly water-soluble drug for respiratory delivery. Chem. Pharm. Bull. 2012, 60, 870-876, https://doi.org/10.1248/cpb.c12-00208.

88. Kundawala, A.J.; Patel, V.A.; Patel, H.V.; Choudhary, D. Treating tuberculosis with chitosan microparticles loaded with rifampicin as respirable powder for pulmonary delivery. Indian J. Nov. Drug Del. 2012, 4, 5765.

89. Mishra, M.; Mishra, B. Mucoadhesive microparticles as potential carriers in inhalation delivery of doxycycline hyclate: a comparative study. Acta Pharm. Sin. B 2012, 2, 518-526, https://doi.org/10.1016/j.apsb.2012.05.001.

90. Yildiz, A.; John, E.; Özsoy, Y.; Araman, A.; Birchall, J.C.; Broadley, K.J.; Gumbleton, M. Inhaled extendedrelease microparticles of heparin elicit improved pulmonary pharmacodynamics against antigen-mediated airway hyper-reactivity and inflammation. J. Controlled Release 2012, 162, 456-463, https://doi.org/10.1016/j.jconrel.2012.07.008.

91. Scalia, S.; Haghi, M.; Losi, V.; Trotta, V.; Young, P.M.; Traini, D. Quercetin solid lipid microparticles: a flavonoid for inhalation lung delivery. Eur. J. Pharm. Sci. 2013, 49, 278-285, https://doi.org/10.1016/j.ejps.2013.03.009.

92. Durli, T.L.; Dimer, F.A.; Fontana, M.C.; Pohlmann, A.R.; Beck, R.C.R.; Guterres, S.S. Innovative approach to produce submicron drug particles by vibrational atomization spray drying: influence of the type of solvent and surfactant. Drug Dev. Ind. Pharm. 2014, 40, 1011-1020,https://doi.org/10.3109/03639045.2013.798804.

93. Daman, Z.; Gilani, K.; Najafabadi, A.R.; Eftekhari, H.R.; Barghi, M.A. Formulation of inhalable lipid-based salbutamol sulfate microparticles by spray drying technique. DARU J. Pharm. Sci. 2014, 22, https://doi.org/10.1186/2008-2231-22-50

94. Beck-Broichsitter, M.; Strehlow, B.; Kissel, T. Direct fractionation of spray-dried polymeric microparticles by inertial impaction. Powder Technol. 2015, 286, 311-317, https://doi.org/10.1016/j.powtec.2015.08.033.

95. Martignoni, I.; Trotta, V.; Lee, W.H.; Loo, C.Y.; Pozzoli, M.; Young, P.M.; Scalia, S.; Traini, D. Resveratrol solid lipid microparticles as dry powder formulation for nasal delivery, characterization and in vitro deposition study. J. Microencapsul. 2016, 33, 735-742, https://doi.org/10.1080/02652048.2016.1260659.

96. Alves, A.D.; Cavaco, J.S.; Guerreiro, F.; Lourenço, J.P.; Rosa da Costa, A.M.; Grenha, A. Inhalable antitubercular therapy mediated by locust bean gum microparticles. Molecules 2016, 21, https://doi.org/10.3390/molecules21060702.

97. Jeong, D.; Kang, C.; Jung, E.; Yoo, D.; Wu, D.; Lee, D. Porous antioxidant polymer microparticles as therapeutic systems for the airway inflammatory diseases. J. Controlled Release 2016, 233, 72-80, https://doi.org/10.1016/j.jconrel.2016.04.039.

98. Shen, Y.B.; Du, Z.; Tang, C.; Guan, Y.X.; Yao, S.J. Formulation of insulin-loaded N-trimethyl chitosan microparticles with improved efficacy for inhalation by supercritical fluid assisted atomization. Int. J. Pharm. 2016, 505, 223-233, https://doi.org/10.1016/j.ijpharm.2016.03.053.

99. Wang, Q.; Mi, G.; Hickey, D.; Li, Y.; Tu, J.; Webster, T.J.; Shen, Y. Azithromycin-loaded respirable microparticles for targeted pulmonary delivery for the treatment of pneumonia. Biomaterials 2018, 160, 107123, https://doi.org/10.1016/j.biomaterials.2018.01.022.

100. Takeuchi, I.; Taniguchi, Y.; Tamura, Y.; Ochiai, K.; Makino, K. Effects of L-leucine on PLGA microparticles for pulmonary administration prepared using spray drying: Fine particle fraction and phagocytotic ratio of alveolar macrophages. Colloid Surface A 2018, 537, 411-417, https://doi.org/10.1016/j.colsurfa.2017.10.047.

101. Ceschan, N.E.; Bucalá, V.; Mateos, M.V.; Smyth, H.D.C.; Ramírez-Rigo, M.V. Carrier free indomethacin microparticles for dry powder inhalation. Int. J. Pharm. 2018, 549, 169-178, https://doi.org/10.1016/j.ijpharm.2018.07.065.

102. Amore, E.; Manca, M.L.; Ferraro, M.; Valenti, D.; La Parola, V.; Di Vincenzo, S.; Gjomarkaj, M.; Giammona, G.; Bondì, M.L.; Pace, E. Salmeterol Xinafoate (SX) loaded into mucoadhesive solid lipid microparticles for COPD treatment. Int. J. Pharm. 2019, 562, 351-358, https://doi.org/10.1016/j.ijpharm.2019.03.059. 
103. Shahin, H.I.; Vinjamuri, B.P.; Mahmoud, A.A.; Shamma, R.N.; Mansour, S.M.; Ammar, H.O.; Ghorab, M.M.; Chougule, M.B.; Chablani, L. Design and evaluation of novel inhalable sildenafil citrate spray-dried microparticles for pulmonary arterial hypertension. J. Control Release 2019, 302, 126-139, https://doi.org/10.1016/j.jconrel.2019.03.029.

104. Zhang, L.; Zhang, X.; Li, J.; Beck-Broichsitter, M.; Muenster, U.; Wang, X.; Zhao, J.; Mao, S. Optimization of budesonide-loaded large-porous microparticles for inhalation using quality by design approach. J. Drug Deliv. Sci. Technol. 2019, 53, 101-140, https://doi.org/10.1016/j.jddst.2019.101140.

105. O'Connor, G.; Krishnan, N.; Fagan-Murphy, A.; Cassidy, J.; O'Leary, S.; Robertson, B.D.; Keane, J.; O'Sullivan, M.P.;Cryan, S.A. Inhalable poly (lactic-co-glycolic acid)(PLGA) microparticles encapsulating all-trans-Retinoic acid (ATRA) as a host-directed, adjunctive treatment for Mycobacterium tuberculosis infection. Eur J Pharm Biopharm. 2019, 134, 153-165, https://doi.org/10.1016/j.ejpb.2018.10.020.

106. Guerreiro, F.; Pontes, J.F.; da Costa, A.M.R.; Grenha, A. Spray-drying of konjac glucomannan to produce microparticles for an application as antitubercular drug carriers. Powder Technol. 2019, 342, 246-252, https://doi.org/10.1016/j.powtec.2018.09.068.

107. Nurbaeti, S.N.; Brillault, J.; Tewes, F.; Olivier, J.C. Sustained-release microparticle dry powders of chloramphenicol palmitate or thiamphenicol palmitate prodrugs for lung delivery as aerosols. Eur. J. Pharm. Sci. 2019, 138, 105-128, https://doi.org/10.1016/j.ejps.2019.105028.

108. Kucukoglu, V.; Uzuner, H.; Kenar, H.; Karadenizli, A. In vitro antibacterial activity of ciprofloxacin loaded chitosan microparticles and their effects on human lung epithelial cells. Int. J. Pharm. 2019, 569, https://doi.org/10.1016/j.ijpharm.2019.118578.

109. Wong, J.T.F.; Tam, M.S.C. Pulmonary drug delivery system. U.S. Patent 005451569A. 1995.

110. Hanes, J.; Edwards, D.A.; Evora, C.; Langer, R. Particles Incorporating Surfactants for pulmonary drug delivery, unit dose microcartridges dry powder, related devices and methods. U.S. Patent 005855913A. 1999.

111. Feldstein, R.; Lian, H.; Rodes, C.A.; Shen, G.S.; Steiner, S.S. Method for drug delivery to the pulmonary system. W.O. Patent 1996036314A3. 1996.

112. Bernstein, H.; Chickering, I.D.E.; Huang, E.K.; Narasimhan, S.; Reese, S.; Straub, J.A. Sustained release porous microparticles for inhalation. W.O. Patent 2004030659A1.2004

113. Sen, H.; Jayanthi, S.; Sinha, R.; Sharma, R.; Muttil, P. Inhalable biodegradable microparticles for targetspecific drug delivery in tuberculosis and a process thereof. U.S. Patent 2005/0084455 A1.2005.

114. Sen, H.; Jayanthi, S.; Sinha, R.; Sharma, R.; Muttil, P. Inhalable Biodegradable microparticle for target specific drug delivery in tuberculosis and a process thereof. U.S. Patent 2007154408. 2007.

115. King, M.; Warden, J.A.; Kim, J.; Finney, B.; Oxley, N. Dry powder inhalers having spiral travel paths. U.S. Patent 20070215149A1. 2007

116. Colombo, P.; Cagnani, S.; Ventura, P. Insulin highly respirable microparticles. U.S. Patent 7625865B2. 2009.

117. Chimote, G.C.; Mahajan, G.B.; Vasudevan, A.; Hariharan, S. Microparticle formulation for pulmonary drug delivery of anti-infective molecule for treatment of infectious disease. W.O. Patent 2012017405A1.2012.

118. Kraft, K.S.; Somerville, K. Diketopiperazine with defined isomer contents. U.S. Patent 8227409B2. 2012.

119. Kumar, A.; Mohapatra, S.S.; Cameron, D.F. Nanoparticles targeted drug delivery to the lungs using extratesticular sertoli cells. U.S. Patent OO916 1901 B2. 2015.

120. Yaadi, K. Dry powder formulations for inhalation W.O. Patent 2016/019253 Al.2016

121. Andrea Leone-Bay Houghten Richard, A.; Guarneri, J.J.; Stowell, G.W. Compositions for treating pain. J.P. Patent 2016222689. 2016.

122. Smutney, C.C.; Adamo, B.; Polidoro, J.M.; Kinsey, P.S.; Overfield, D.; Sahi, C.R.; Billings, C.; Marino, M.T. Dry powder drug delivery system. U.S. Patent 10342938B2. 2019.

123. Swarbrick, J. Encyclopedia of Pharmaceutical Technology. Informa Healthcare, Third Edition 2007.

124. Harrison, E. National Respiratory Care Handbook. Jones and Bartlett Publishers, 2011.

125. Pfützner, A.; Mann, A.E.; Steiner, S.S. Technosphere ${ }^{\mathrm{TM}} /$ Insulin-a new approach for effective delivery of human insulin via the pulmonary route. Diabetes Technol. Ther. 2002, 4, 589-594, https://doi.org/10.1089/152091502320798204.

126. https://www.pharmaceuticalonline.com/doc/pfizer-receives-fda-approval-for-exubera-the-0001

127. Lipworth, B.J.; Sims, E.J.; Taylor, K.; Cockburn, W.; Fishman, R. Dose-response to salbutamol via a novel palm sized nebuliser (Aerodose ${ }^{\circledR}$ Inhaler), conventional nebuliser (Pari LC Plus) and metered dose inhaler (Ventolin $^{\mathrm{TM}}$ Evohaler ${ }^{\mathrm{TM}}$ ) in moderate to severe asthmatics. Br. J. Clin. Pharmacol. 2005, 59, 5-13, https://doi.org/10.1111/j.1365-2125.2005.02168.x.

128. Cefalu, W.T.; Skyler, J.S.; Kourides, I.A.; Landschulz, W.H.; Balagtas, C.C.; Cheng, S.L.; Gelfand, R.A. Inhaled human insulin treatment in patients with type 2 diabetes mellitus. Ann. Intern. Med. 2001, 134, 203207, https://doi.org/10.7326/0003-4819-134-3-200102060-00011.

129. Perera, A.D.; Kapitza, C.; Nosek, L.; Fishman, R.S.; Shapiro, D.A.; Heise, T.; Heinemann, L. Absorption and metabolic effect of inhaled insulin: intrapatient variability after inhalation via the Aerodose insulin inhaler in patients with type 2 diabetes. Diabetes Care 2002, 25, 2276-2281, https://doi.org/10.2337/diacare.25.12.2276. 
130. Kim, D.; Mudaliar, S.; Chinnapongse, S.; Chu, N.; Boies, S.M.; Davis, T.; Perera, A.D.; Fishman, R.S.; Shapiro, D.A.; Henry, R. Dose-response relationships of inhaled insulin delivered via the Aerodose insulin inhaler and subcutaneously injected insulin in patients with type 2 diabetes. Diabetes Care 2003, 26, 28422847, https://doi.org/10.2337/diacare.26.10.2842.

131. Rave, K.; Nosek, L.; Heinemann, L.; Gonzales, C.; Ernest, C.S.; Chien, J.; Muchmore, D. Inhaled micronized crystalline human insulin using a dry powder inhaler: dose-response and time-action profiles. Diabet. Med. 2004, 21, 763-768, https://doi.org/10.1111/j.1464-5491.2004.01240.x.

132. Schuster, J.; Rubsamen, R.; Lloyd, P.; Lloyd, J. The AERx ${ }^{\text {TM }}$ aerosol delivery system. Pharmaceutical Res. 1997, 14, 354-357, https://doi.org/10.1023/a:1012058323754.

133. Farr, S.J.; McElduff, A.; Mather, L.E.; Okikawa, J.; Ward, M.E.; Gonda, I.; Licko, V.; Rubsamen, R.M. Pulmonary insulin administration using the AERx® system: physiological and physicochemical factors influencing insulin effectiveness in healthy fasting subjects. Diabetes Technol. Ther. 2000, 2, 185-197, https://doi.org/10.1089/15209150050025131. 